\title{
Impacts of Boundary Conditions on Premixed Combustion in Obstructed Conduits: A Computational Analysis
}

\author{
Rawan Ibrahim Alkandari
}

Follow this and additional works at: https://researchrepository.wvu.edu/etd

\section{Recommended Citation}

Alkandari, Rawan Ibrahim, "Impacts of Boundary Conditions on Premixed Combustion in Obstructed Conduits: A Computational Analysis" (2017). Graduate Theses, Dissertations, and Problem Reports. 5071. https://researchrepository.wvu.edu/etd/5071

This Thesis is protected by copyright and/or related rights. It has been brought to you by the The Research Repository @ WVU with permission from the rights-holder(s). You are free to use this Thesis in any way that is permitted by the copyright and related rights legislation that applies to your use. For other uses you must obtain permission from the rights-holder(s) directly, unless additional rights are indicated by a Creative Commons license in the record and/ or on the work itself. This Thesis has been accepted for inclusion in WVU Graduate Theses, Dissertations, and Problem Reports collection by an authorized administrator of The Research Repository @ WVU. For more information, please contact researchrepository@mail.wvu.edu. 


\title{
Impacts of Boundary Conditions on Premixed Combustion in Obstructed Conduits: A Computational Analysis
}

\author{
Rawan Ibrahim Alkandari \\ Thesis submitted to the \\ Benjamin M. Statler College of Engineering and Mineral Resources \\ at West Virginia University \\ in partial fulfillment of the requirements for the degree of \\ Master of Science \\ in Mechanical Engineering \\ V'yacheslav Akkerman, Ph.D., Chair \\ Terence Musho, Ph.D. \\ Osama Mukdadi, Ph.D. \\ Department of Mechanical and Aerospace Engineering
}

Morgantown, West Virginia

2017

Keywords: Premixed, Obstructed conduits, Wall friction, Boundary conditions, Flame Oscillations, Flame acceleration, Computational simulations

Copyright 2017 Rawan Ibrahim Alkandari 


\section{ABSTRACT \\ Impacts of Boundary Conditions on Premixed Combustion in Obstructed Conduits: A Computational Analysis}

\section{Rawan Ibrahim Alkandari}

Premixed flame acceleration in channels or pipes has various practical applications, starting with fire safety problems and ending with advanced technologies such as pulse-detonation engines. In particular, a flame accelerates extremely fast when propagating through a comb-shaped array of narrow, tightlyspaced obstacles in a so-called "Bychkov tube". In the present thesis, the role of boundary conditions in such geometry is studied by means of the comprehensive computational simulations of combustion equations, with a fully-compressible hydrodynamics and an Arrhenius chemical kinetics. Specifically, the mechanistic (slip/nonslip) and thermal (adiabatic/isothermal) conditions at the walls/obstacles' surfaces, as well as the boundaries at the conduits' extremes (open/closed) are considered. The parametric study includes: the thermal expansion ratio in the burning process $\Theta=\rho_{\text {unburnt }} / \rho_{\text {burnt }}$ in the range $5 \leq \Theta \leq 10$; the wall temperature $T_{w}$ being $298 \mathrm{~K} \leq T_{w} \leq 1000 \mathrm{~K}$; the pipe radius $R$ exceeding the thermal flame thickness $L_{f}$ by a factor of 12 48; the obstacles blockage ratio $\alpha$ in the range $1 / 3 \leq \alpha \leq 2 / 3$; and the spacing between the obstacles $\Delta \mathrm{z}$ being $0.25 R \leq \Delta \mathrm{z} \leq 2 R$.

It is shown that the impacts of both mechanistic and thermal surface conditions on flame propagation are minor and can be omitted. This is because the flame dynamics if mainly driven by flame spreading in an unobstructed portion of an obstructed pipe, i.e. far from the walls. With a fact that real walls are neither slip nor nonslip; neither adiabatic nor isothermal, but in between these categories, the minor role of surface conditions, identified here, validates the Bychkov model, which employs a number of simplifying assumptions, including slip and adiabatic surfaces.

In contrast, the role of the conditions at a pipe extreme is shown to be substantial. While in a semi-open pipe (one end is closed; a flame is ignited at this end and propagates towards the open end), the entire flame-generated jet-flow is pushed towards a single exit, in a pipe with two ends open, this flow is distributed between the upstream and downstream flows, thereby moderating flame propagation. As a result, in this geometry, a flame either accelerates much weaker (in a relatively wide pipe), with a possibility of blowout, or oscillates (in a narrow pipe). The oscillations appear nonlinear in all the situations when they are observed, and the present thesis quantifies the oscillation period and amplitude as well as the average flame velocity in the $\Theta-T_{w^{-}}-\alpha-\Delta \mathrm{z}-R$ space.

Since these flame oscillations can be treated as fluctuations around a quasi-steady solution, the present thesis qualitatively supports the recent experiments, modeling and theory of flames in obstructed pipes with both ends open, which all yielded steady or quasi-steady flame propagation prior to an onset of spontaneous flame acceleration and deflagration-to-detonation transition. 


\section{Dedication}

I dedicate this work to my family and to those who inspired me to seek knowledge. 


\section{Acknowledgements}

This thesis would not have been possible without those individuals who helped me put this work together.

First, I own my deepest gratitude to my supervisor, Dr. V'yacheslav Akkerman, who guided me from the start until the end of this project. I want to thank him for supporting me and for being patient. This work would not be possible without him. It is an honor for me to work with such an amazing and kind professor.

I would like to thank my committee members as well for supporting me and helping me during my undergraduate and graduate years. I learned a lot from both Dr. Musho and Dr. Mukdadi.

A special thanks to my colleagues, Sinan Demir, Abdulafeez Adebiyi, Gbolahan Idowu, Swaithi Shetty, Furkan Kodakoglu, Elizabeth Ridgeway and Amanda Cathreno who have helped me with my computational analysis and results.

Lastly, I would like to thank my family and friends for their unconditional love and support. 


\section{Table of Contents}

ABSTRACT 1..............................................................................................................ii

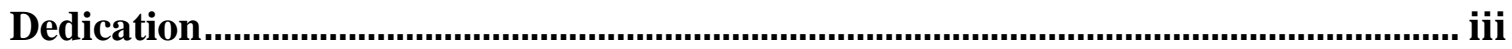

Acknowledgements.........................................................................................................

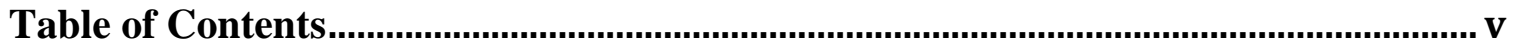

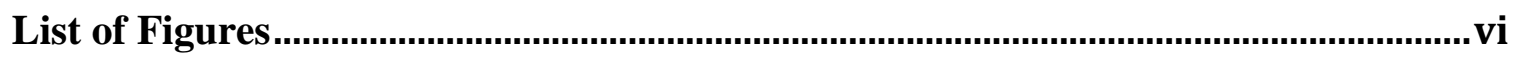

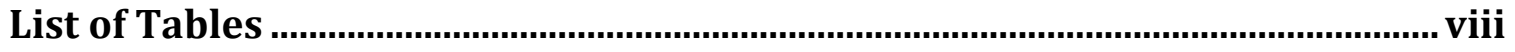

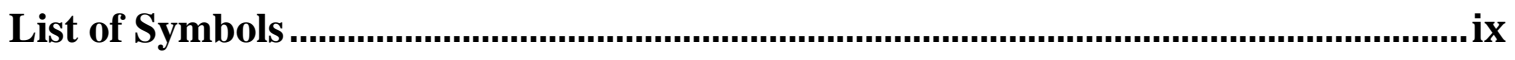

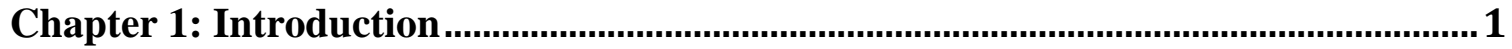

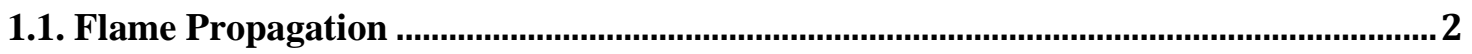

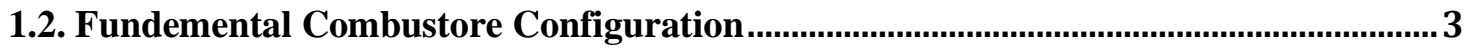

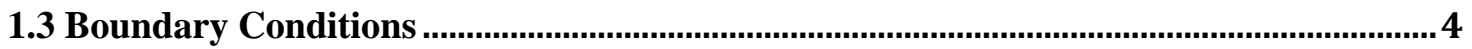

1.4 Unobstructed Vs Obstructed Conduits...................................................................... 5

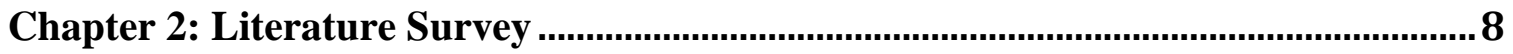

2.1. Flame Acceleration in Semi-Open Obstructed Channels.................................................8

2.2. Flame Osillation/Acceleration in Fully Open Obstructed Channels ........................... 12

Chapter 3: Numerical Method .............................................................................. 15

Chapter 4: Results and Discussion ....................................................................... 19

4.1. Flame Acceleration in Semi-Open Obstructed Channels............................................ 19

4.2. Flame Oscillation/Acceleration in Fully Open Obstructed Channels .......................... 26

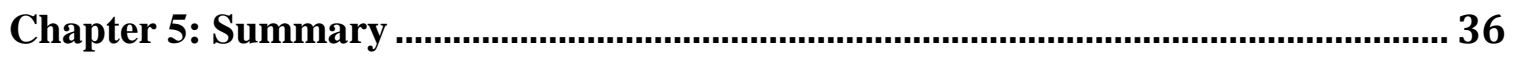

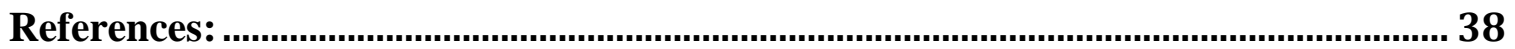




\section{List of Figures}

Figure 1.1 A schematic of a semi-open unobstructed channel (a), an open unobstructed channel (b), a semi-open obstructed channel $(\boldsymbol{c})$ and an open obstructed channel $(\boldsymbol{d}) \ldots . . .7$

Figure 2.1 Illustration of the physical mechanism of ultrafast flame acceleration in semiopen channels with obstacles.

Figure 2.2 Numerical simulations of the toothbrush set of obstacles (snapshot). .10

Figure 2.3 A Hemispherical flame initiated from the closed end ...........................12

Figure 2.4 Schematic of an open-open obstructed channel...........................13

Figure 4.1 The scaled flame position $Z_{f} / R$ vs the scaled time $\tau$ for $R=24 L_{f}, \alpha=1 / 3$ and $\Delta Z / R=1 / 4$.

Figure 4.2 The scaled flame position $Z_{f} / R$ vs the scaled time $\tau$ for $R=24 L_{f}, \alpha=1 / 3,1 / 2$, $2 / 3$ and $\Delta Z / R=1 / 4$ .20

Figure 4.3 The scaled flame position $Z_{f} / R$ vs the scaled time $\tau$ for $R=24 L_{f}, \alpha=2 / 3$ and $\Delta Z / R=1 / 2,1 / 4$. 20

Figure 4.4 The flame evolution in tube of $R=24 L_{f}, \alpha=2 / 3$ and $\Delta Z / R=1 / 2$ : a) slip and $b$ ) no-slip.

Figure 4.5 The scaled flame position $Z_{f} / R$ vs the scaled time $\tau$ for $R=24 L_{f}, \alpha=2 / 3$ and $\Delta Z / R=1 / 4,1 / 2,1$. 22

Figure 4.6 The scaled flame position $Z_{f} / R$ vs the scaled time $\tau$ for $R=12 L_{f}, \alpha=1 / 3$ and $\Delta Z / R=1 / 4,1 / 2,1$.

Figure 4.7 The scaled flame position $Z_{f} / R$ vs the scaled time $\tau$ for $R=36 L_{f}, \alpha=1 / 3$ and $\Delta Z / R=1$.

Figure 4.8 The flame evolution in tube of $R=36 L_{f}, \alpha=1 / 3$ and $\Delta Z / R=1$ : a) slip and $b$ ) noslip.

Figure 4.9 The flame evolution in tube of $R=36 L_{f}, \alpha=1 / 2$ and $\Delta Z / R=1$ : a) slip and $b$ ) noslip.

Figure 4.10 The flame evolution in tube of $R=36 L_{f}, \alpha=2 / 3$ and $\triangle Z / R=1$ : a) slip and $b$ ) noslip.....

Figure 4.11 The scaled flame position $Z_{f} / R$ vs the scaled time $\tau$ for $R=12 L_{f}, \alpha=1 / 3$ and $\Delta Z / R=2$.

Figure 4.12 The flame evolution in tube of $R=12 L_{f}, \alpha=1 / 3$ and $\triangle Z / R=2$ : a) slip and $b$ ) noslip. 
Figure 4.13 The scaled flame position $U_{t} / S_{L}$ vs the scaled time $\tau$ for $R=20 L_{f}, \alpha=1 / 2$ and $\Delta Z / R=0.2[17]$

Figure 4.14 The scaled flame position $U_{t} / S_{L}$ vs the scaled time $\tau$ for $R=24 L_{f}$, and $\Delta Z / R=$ 0.2 at different $\alpha$ [17].

Figure 4.15 The flame evolution in tube of $R=20 L_{f}, \alpha=1 / 2$ and $\Delta Z / R=0.2$ for adiabatic walls/obstacles [17]. .26

Figure 4.16 The flame evolution in tube of $R=20 L_{f}, \alpha=1 / 2$ and $\Delta Z / R=0.2$ for isothermal (1000K) walls/obstacles [17]..... .26

Figure 4.17 Evolution of the flame and the flow in an obstructed channel of $R=12 L_{f}$ and $\alpha=1 / 3$.

Figure 4.18 Evolution of the flame and the flow in an obstructed channel of $R=12 L_{f}$ and $\alpha=1 / 2$. 27

Figure 4.19 Evolution of the flame and the flow in an obstructed channel of $R=12 L_{f}$ and $\alpha=2 / 3$. .27

Figure 4.20 The scaled burning rate $U_{t} / S_{L}$ vs the scaled time $\tau$ for $R / L_{f}=12$ and $\alpha=1 / 2$, $1 / 3,2 / 3, \Delta Z / R=1 / 4$. 29

Figure 4.21 The scaled burning rate $U_{t} / S_{L}$ vs the scaled time $\tau$ for $R / L_{f}=24$ and $\alpha=1 / 2$, $2 / 3, \Delta Z / R=1 / 4$. 29

Figure 4.22 The scaled burning rate $U_{w} / S_{L}$ vs the scaled time $t S_{L} / R$ for the thermal expansion ratio $\theta=8$, the blockage ratio $\alpha=1 / 3$ and various obstacle spacing $\Delta Z / R=$ 0.25 , blue; $\Delta Z / R=0.5$, red; and $\Delta Z / R=1$, black.

Figure 4.23 The scaled burning rate $U_{w} / S_{L}$ vs the scaled time $t S_{L} / R$ for $\Delta Z / R=0.5$ and blockage ratios $\alpha=1 / 3$; with thermal expansions $\theta=5$, blue; $\theta=8$, red; and $\theta=10$, black.

Figure 4.24 The scaled burning rate $U_{w} / S_{L} v$ s the scaled time $t S_{L} / R$ for $\Delta Z / R=0.5$ and blockage ratios $\alpha=1 / 2$; with thermal expansions $\theta=5$, blue; $\theta=8$, red; and $\theta=10$, black....

Figure 4.25 The scaled burning rate $U_{w} / S_{L}$ vs the scaled time $t S_{L} / R$ for $\Delta Z / R=0.5$ and blockage ratios $\alpha=2 / 3$; with thermal expansions $\theta=5$, blue; $\theta=8$, red; and $\theta=10$, black.

Figure 4.26 The scaled oscillation period vs the thermal expansion ratio $\theta$ for various blockage ratios: $\alpha=1 / 3$ (blue); $\alpha=1 / 2$ (red); and $\alpha=2 / 3$ (black)....................... 32

Figure 4.27 The scaled burning rate averaged over an oscillation period vs the thermal expansion ratio $\theta$ for various blockage ratios: $\alpha=1 / 3$ (blue); $\alpha=1 / 2$ (red); and $\alpha=2 / 3$ (black). 
Figure 4.28 The oscillation amplitude vs the thermal expansion ratio $\theta$ for various blockage ratios: $\alpha=1 / 3$ (blue); $\alpha=1 / 2$ (red); and $\alpha=2 / 3$ (black)........................33

Figure 4.29 The scaled flame position vs the scaled time: a) $R=12 L_{f}, \alpha=1 / 2,2 / 3$ and $\Delta Z / R=1 / 4 . b$ ) recent experiment [24], modeling [23,24] and theory [18] .................34

Figure 4.30 The scaled burning rate $U_{t} / S_{L}$ vs the scaled time $\tau$ for $R / L f=24$ and $\Delta Z / R=$

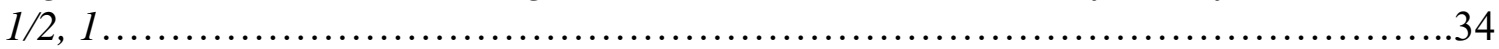

Figure 4.31 The scaled burning rate $U_{t} / S_{L}$ vs the scaled time $\tau$ for $R / L_{f}=36,48$;

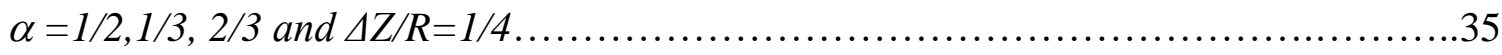

\section{List of Tables}

Table 1.1 Fundamental Combustors configuration....................................... 


\section{List of Symbols}

$\Delta z$

$I_{0}$

$L$

$P_{1}$

$P_{2}$

$P_{b}$

$P_{f}$

$R$

$\operatorname{Re}$

$S_{L}$

$U_{1}$

$U_{2}$

$U_{f}$

CJ

$\mathrm{Cp}$

$\mathrm{Cv}$

DDT

$\mathrm{D}_{\text {th }}$

$\mathrm{E}_{\mathrm{A}}$

FA

Le

$L_{f}$

$\mathrm{m}$

$\mathrm{Ma}$

$\mathrm{n}$

Pr

Q

$\mathrm{q}_{\mathrm{i}}$

$\mathrm{r}_{\mathrm{f}}$

$\mathrm{Rp}$

Sc

$\mathrm{T}$

Tw

u

Y

$\mathrm{Z}$

$\mathrm{Ze}$
Spacing between obstacles

Modified Bessel function of order zero

Length of the channel

Pressure at entrance of the channel

Pressure at the exit of the channel

Pressure of burned fuel

Pressure of fresh fuel mixture

Radius

Flame propagation Reynolds number

Laminar flame speed

Velocity at channel entrance

Velocity at channel exit

Laminar flame speed

Chapman-Jouget

Heat capacity at constant pressure

Heat capacity at constant volume

Deflagration to Detonation Transition

Thermal diffusion coefficient

Activation energy

Flame acceleration

Lewis number

Flame thickness

Constant molar mass

Mach number

Normal vector

Prandtl number

Specific energy released

Energy diffusion

Flame radius

Universal gas constant

Schmidt number

Temperature

Wall temperature

Velocity component

Mass fraction of fuel

Axial flame position

Zeldovich number 


$\begin{array}{ll}\text { GREEK: } & \\ \alpha & \text { Thermal expansion coefficient } \\ & \text { Blockage ratio } \\ & \text { Viscosity } \\ & \text { Dynamic viscosity coefficient } \\ \mu & \text { Dynamic viscosity coefficient in the fuel } \\ \mu_{\mathrm{f}} & \text { Flame speed variable } \\ \beta & \text { Adiabatic exponent } \\ \gamma & \text { Stress tensor } \\ \zeta & \text { Density } \\ \rho & \text { Burned gas density } \\ \rho_{\mathrm{b}} & \text { Fresh gas mixture density } \\ \rho_{\mathrm{f}} & \text { Acceleration rate } \\ \sigma & \text { Scaled time } \\ \tau & \text { Total energy per unit volume } \\ \varepsilon & \end{array}$




\section{Chapter 1: Introduction}

Combustion is an exothermic chemical reaction between a combustible fuel and an oxidant. The former is oxidized by the latter, most commonly atmospheric oxygen, resulting in a release of energy in the form of heat because of breaking of bonds in the fuel. When heat transfer and free radicals (active species) initiate a chemical reaction within the adjacent layer of the combustible mixture, this layer turns to be the source of the radicals as well as the heat source. Consequently, the layer becomes capable of initiating a chemical reaction in the adjacent/next layer.

In a particular case of hydrocarbon oxy-combustion, the products released at the end of the reaction are carbon dioxide $\left(\mathrm{CO}_{2}\right)$ and water $\left(\mathrm{H}_{2} \mathrm{O}\right)$ vapor, even though other minor intermediate radicals such as carbon monoxide $(\mathrm{CO})$ are also released during the process; this depends on the composition of the reactant among a myriad of other factors. Combustion can either be classified as complete or incomplete. In a complete combustion process, the reactant is completely burned in oxygen producing a given number of products which is limited. For instance, in the example mentioned above, if a hydrocarbon is burned through oxidation, the end products are water and carbon dioxide - therefore, complete combustion mostly produces oxides as the final products. Incomplete combustion occurs when a reaction does not have a sufficient amount of oxygen thereby producing water and carbon monoxide. Combustion can also be hindered by heat sink leading to incomplete combustion of the reactant [1].

The general formula for a hydrocarbon oxy-combustion reaction reads

$$
\mathrm{C}_{\mathrm{X}} \mathrm{H}_{\mathrm{Y}}+\mathrm{O}_{2} \longrightarrow \mathrm{CO}_{2}+\mathrm{H}_{2} \mathrm{O}
$$

Moreover, the standard classification of combustion regimes includes diffusion (non-premixed) and premixed burning. Diffusion combustion means that the fuel and oxidizer are not mixed prior to the reacting meaning that the fuel and oxidizer are carried separately into the burning zone. Lighting a candle is an example of diffusion combustion. Premixed combustion occurs when the fuel and oxidizer are already mixed before the ignition. A gas stove is an example of premixed burning. It is noted that premixed combustion may proceed in two regimes, that is the deflagration or flame (subsonic regime) and the detonation (supersonic regime) [2, 3, 4].

- Deflagration: the reaction propagates due to the thermal conduction, with a speed of the order of $1 \mathrm{~m} / \mathrm{s}$ such that a Mach number $\mathrm{Ma}<<1$. It can be seen in daily life applications 
such as internal combustion engines, fireworks and gunpowder [3, 4] since deflagrations are controllable.

- Detonation: the reaction is driven by the shockwaves, with a speed of the order of $10^{3} \mathrm{~m} / \mathrm{s}$ and $\mathrm{Ma}>1$. A high explosive like dynamite can be considered as detonation [3, 4].

Additionally, a flame may accelerate spontaneously, resulting eventually into a deflagration-todetonation transition (DDT) event $[3,4]$.

Combustion has both domestic and industrial applications which make the process useful. In the domestic scenario, it is through combustion that heat energy is produced for a variety of purposes such as cooking, heating, and so on. Combustion mechanisms are also used in industries, for example premixed flames are put under pressure to necessitate the production of final products in industrial plants as well as to study the fundamentals of flame acceleration (FA) and DDT. Additionally, they are also used to mitigate or avoid fire safety hazards and to facilitate combustion in novel energy-efficient devices such as pulse-detonation engines (PDE), rotationdetonation engines (RDE), and micro-combustors.

\subsection{Flame propagation}

One of the subjects investigated within the frame of combustion science is flame propagation. The latter refers to spreading of a "combustion wave" or a reaction zone through the combustible mixture [5]. Combustion and flame propagation most of the time occur concurrently when a combustible fuel is introduced to high temperatures. The theory referred to as the quantitative theory of propagation is essentially based on mass and heat transfer from the zone of reaction to the mixture which is not burned. The rise of enthalpy across the burning zone as a result of a combustion process is well-balanced through heat conduction from the reaction zone. The flame front propagates with a velocity which is determined by: the type of fuel, the oxidant-fuel mass ratio, the original temperature of the combustible mixture, pressure, the pattern of flow, and the systems geometry [6].

In a few words, combustion and flame propagation is the process of heat flow whereby an unburnt gas or combustible material is raised to burning or ignition through increase in temperature, as implied by the quantitative theory of propagation. Generally, flames propagate 
differently in different scenarios. There are combustible materials that have a mild flame while others that have explosive flames. In such explosive flames propagation is enabled by turbulent exchange, which might include heterogeneous explosion [5]. In furnaces with full flames propagation is enabled through radiation which might be initiated by prior pyrolysis whereas, in furnaces with mild flames, propagation is done by upstream diffusion and downstream pyrolysis.

\subsection{Fundamental Combustors Configurations}

There are four important fundamental combustors configurations as detailed in Table 1. Specifically, these are combustion tube (a) [7], free space (b) [8], jet (c) and confinement (d) [4]. Table 1 also illustrates the dominant mechanism of flame corrugation/acceleration for each configuration. In our study, we focus on flames in tubes, for multiple reasons. Firstly, as tubes have simple geometry, the basic understanding, theory and approach for simulation is simplified when studying flames in tubes. Secondly, the long tubes have high aspect ratio which delays the detonation thereby allowing a longer time period to study flame propagation in them.

\section{Table 1.1 Fundamental combustors configuration}

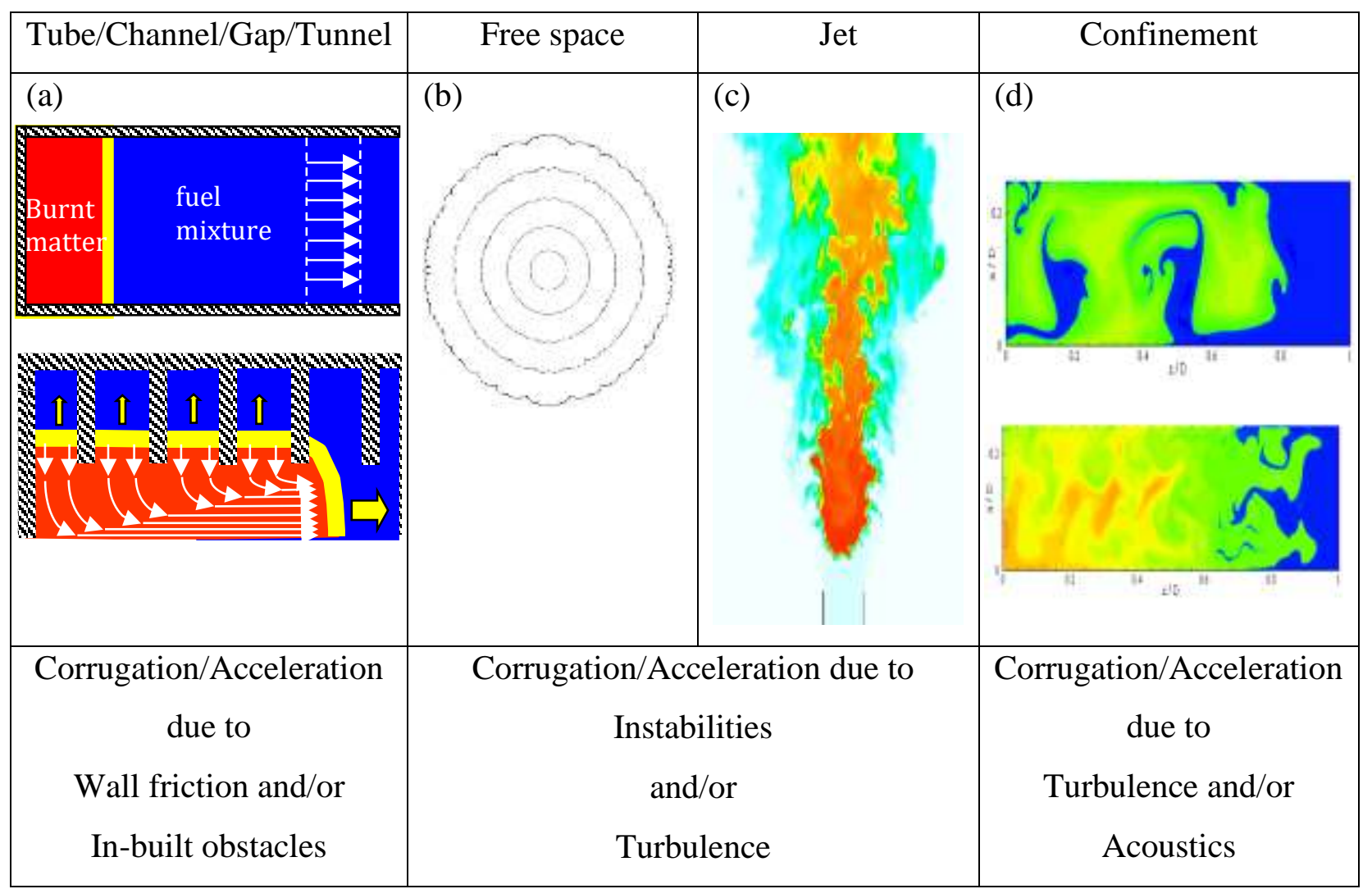


A shape of a flame in a tube has a circular cross section and be elongated along the tube direction. Tubular combustion may be stable since it has thermal and aerodynamic benefits [9].

Tubular flames are prone to spontaneous acceleration, explosion and subsequent detonation as they propagate along the conduit. The acceleration results from the flame becoming non-uniform due to no-slip (frictional) boundary condition, acoustics or turbulence. Non-uniformity distorts the shapre of the flame, which inflates the burning rate and causes the flame to accelerate to detonation. Earlier models of this effect were based mostly on turbulence but later theories and experiments have shown that turbulence is not necessary needed for flame acceleration to occur as the phenomenon has been observed in tubular structures with smooth adiabatic walls [7, 10].

Despite the observation that flame acceleration is stronger in the tubular conduits than in the planar ones, tubular combustion is sometimes not efficient to achieve the DDT because of initial exponential acceleration slowing down due to compression of the gas. But, studies show that adding obstructions along the length of a tubular conduit results in an increase of the flame acceleration rate, leading subsequently to explosion and detonation [7].

\subsection{Boundary conditions}

The mechanistic wall boundary conditions include:

- Slip walls: refer to the symmetrical condition where the wall has no effect on the velocity of the flow close and parallel to the wall.

- Non-slip walls: the condition where the viscous fluid has zero velocity at the wall due to the present adhesive forces between fluid particles and the wall [10].

The thermal walls boundary conditions describe the thermal behavior of a system at its boundary with the surrounding. The thermal boundary conditions include:

- Adiabatic walls: refer to situations where there is no heat transfer between the system and the surrounding across the boundary, and the energy changes in the system are a result of the volumetric change, that is: expansion or contraction. Processes with an adiabatic boundary condition include frictionless compression of gas, insulated fluid flow, and distribution of acoustic waves. 
- Isothermal walls: imply that the temperature at a boundary is fixed despite heat transfer between the system and its surroundings. Instances of isothermal boundary conditions include a solid surface in contact with a boiling liquid [10].

Extremes refer to the nature of the end from which or towards which the flame is propagating. If an end is open, then the shock waves generated by the flame are not reflected back and are dissipated. If an end is closed, then the shock waves are reflected back which creates turbulence and fuel vortexes, therefore, enhancing flame acceleration. In the vented condition, the reflection is interrupted periodically and the acceleration is less than that for the closed system [11]. In our study we investigate semi-open and fully open (both ends open) channels at different parameters.

\subsection{Unobstructed vs Obstructed Conduits}

Unobstructed pipes in semi-open channels [12] (Fig. 1.1a) contain the mechanistic and thermal wall conditions that influence the flame dynamics conceptually. A flame accelerates due to wall friction in an unobstructed, semi-open channel [13], because the flame shape and velocity vary due to flame-wall interactions, but this acceleration is weaker than that with obstructed walls.

The situation is different in unobstructed fully-open channels [12] (Fig. 1.1b). Here the flamegenerated flow is distributed between the upstream and downstream flows, in an intriguing nonlinear manner, and this leads to pulsations of the flame shape and velocity. These pulsations depend on the width of the channel, i.e. when the channels are narrow; the pulsations are rather weak, while in wider channels they are stronger with well-pronounced nonlinear effects.

In the absence of obstacles in channels, experiments have shown that flame acceleration reduces with an increase in a tube diameter. To increase the burning rate and enhance flame acceleration and subsequent detonation, artificial obstacles are placed inside the tube. Aside from these obstacles, producing turbulence, they also create fuel and oxidant mixture pockets between the obstacles thereby improving flame acceleration. The pockets combust later resulting in gas expansion. This causes a strong jet to flow in the unobstructed part of the tube, thus causing faster flame propagation. The increased flame velocity causes an increase in a number of fueloxidant pockets involved, thereby resulting in a positive feedback loop between the flame and 
the burner. Flame acceleration is thus spurred on to supersonic speed to trigger explosion and subsequent detonation. This obstacles-based acceleration does not rely on Reynolds number [7].

In an experimental study of the effect of obstacles with varying blockage ratios on the DDT scenario, the importance of turbulence and obstructions on flame development was observed [14]. The experiments involved physical setups and simulations to observe the flame-tip under different conditions. It was found that the impact of the width of the obstructed tube to the flame acceleration reduced as the flame propagated. Flame acceleration at higher speeds was mainly due to the shock waves from obstructions [14].

It is noted that the obstacles create strong turbulence that is instrumental in increasing the rate of combustion, which expedites flame acceleration. The obstacles provide a unique physical flame propagation mechanism that is unlike acceleration due to wall friction in terms of flame speeds.

In this thesis the following mechanisms were investagated:

* Obstructed semi-open channels (Fig. 1.1c): When a flame propagates in a semi-open channel, its spreading is affected by delayed burning in the pockets causing a flame to accelerate extremely fast in an unobstructed part. While flame propagation through obstacles is usually associated with turbulence/shocks or hydraulic resistance, there is a conceptually laminar, shock-less ultrafast acceleration in semi-open channels as revealed by Bychkov et al. [15]. Obstacles in an enclosure generate acoustic waves which when interact with the flame front thereby promoting flame acceleration.

* Obstructed channels with both ends open (Fig. 1.1d): This mechanism is different from that for semi-open obstructed channels, but the flame acts similar to the unobstructed fully open channels case. It was found that the oscillations of the burning rate and nonlinearity increases with an increase in blockage ratio $\alpha$. 

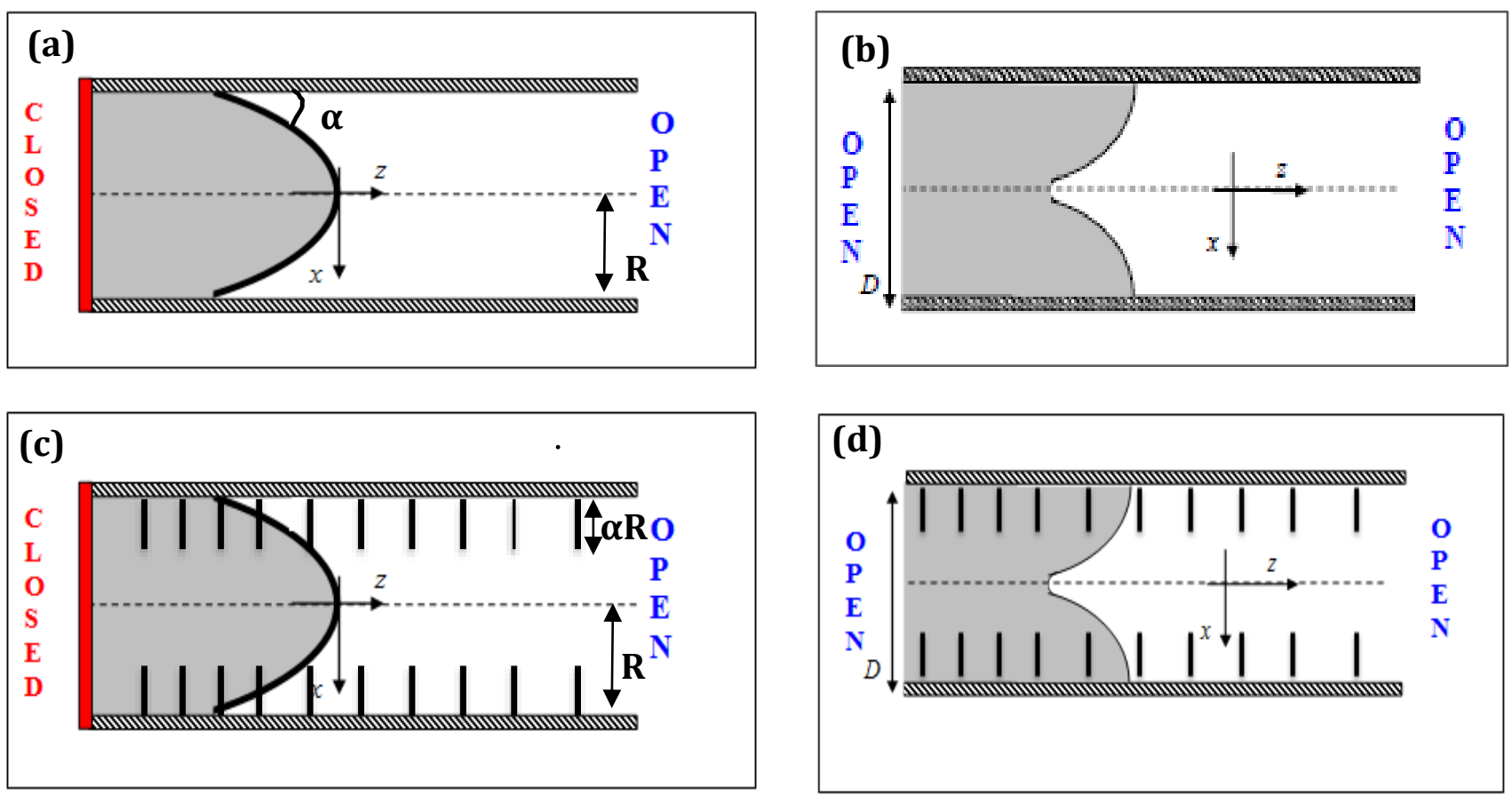

Figure 1.1 A schematic of a semi-open unobstructed channel (a), an open unobstructed channel (b), a semi-open obstructed channel (c) and an open obstructed channel (d). 


\section{Chapter 2: Literature Survey}

\subsection{Flame Acceleration in Semi-Open Obstructed Channels}

According to the Shelkin mechanism, a slow premixed flame accelerates spontaneously due to wall friction from the closed end and triggers detonation [15]. Specifically, the burned gas expands and generates a flow of the fuel mixture. The flow develops turbulence because of the non-slip boundary condition at the wall. Turbulence, in turn, distorts a flame front, hence increasing the burning rate, and it leads to the acceleration. An accelerating flame front pushes compression waves that continuously heat the fuel mixture ahead of it until an explosion is triggered which eventually develops into a detonation [16].

However, it has been later proven that flame acceleration does not only rely on turbulence as Shelkin had explained. Namely, Bychkov et al. [7] have theoretically shown that obstructions affect the rate of acceleration too. Namely, there can be flame acceleration even in the absence of turbulence. Through experiments in smooth micro-tubes, this theory has been validated and, in the end, it also proved that laminar flame acceleration becomes quite weak in wide tubes as Reynolds number of the flow increase. Obstacles placed in the containers are an essential factor in overcoming the heat loss and consequently support the DDT. We assume that the barriers generate stronger turbulence which increases the burning rate and accelerates the flame.

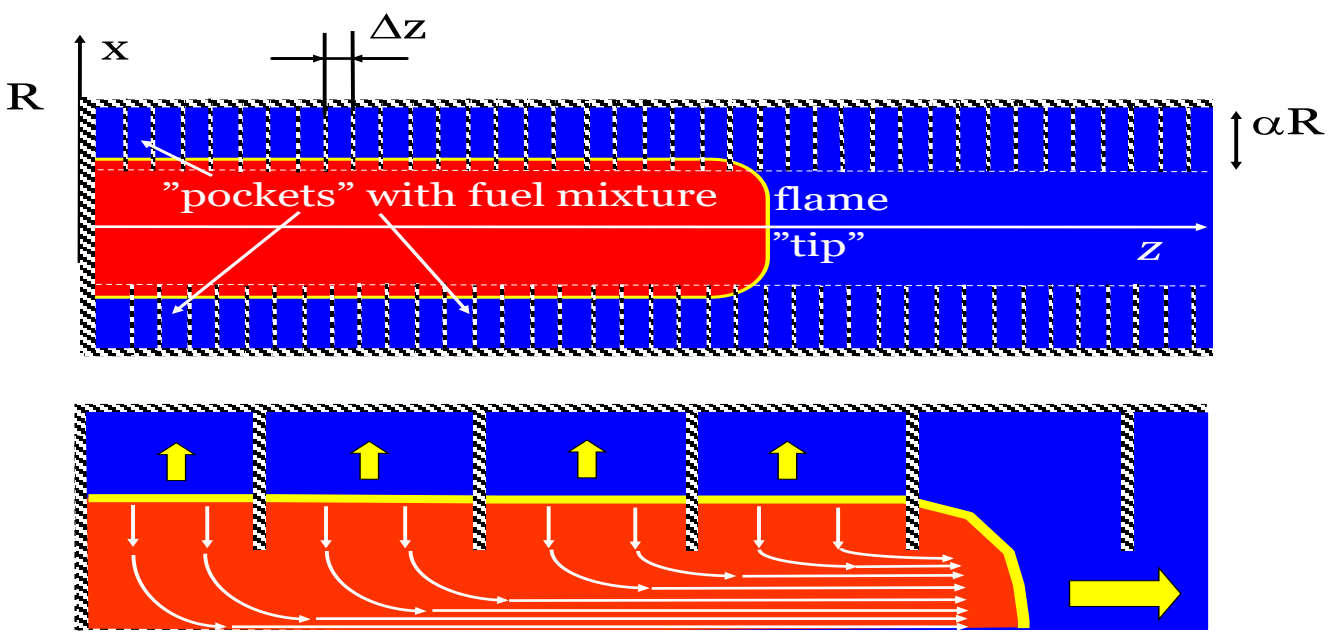

Figure 2.1 Illustration of the physical mechanism of ultrafast flame acceleration in semiopen channels with obstacles [7]. 
The Bychkov mechanism is more robust and provides flame acceleration that is independent of the Reynolds number, therefore, can be necessary for various technical uses and applications. Figure 2.1 illustrates the Bychkov mechanism [7].

In an obstructed channel, there are pockets of a fresh air-fuel mixture that are created between the obstacles, which are initially avoided by the flame front. As a result of delayed burning, there is a gas expansion that produces a powerful jet flow on the unobstructed part of the channel. The jet flow quickens the rate at which the flow propagates, and this generates new pockets that lead to increase in acceleration. Thus, the flame reaches a very high speed with respect to the tube wall, thereby achieving explosion and detonation.

It is also evident from various experiments that flames move faster in cylindrical tubes than in planar channels and this is a result of the change in gas compression [15]. It is also good to note that as the Mach number of the flow increases the acceleration process till it saturates to a statistically constant flame propagation speed. It attains supersonic speed with respect to the tube wall. The saturation state may be reached even before an explosion and subsequent detonation occurs. The flame speed is corrected with the Chapman-Jouguet (CJ) deflagration speed and the state of fast flames observed experimentally. Then it is now possible to show numerically that flame acceleration may lead to explosion and trigger detonation [15].

When the flame propagates from the closed end of the semi-infinite channel in which a fraction of the tube is obstructed, it propagates extremely fast along the unobstructed part. It leaves pockets behind the unburned mixture, between the obstacles which are to burn later. The pockets act as mini-channels in which the flame can be assumed to propagate mainly in the radial direction. It is important to note that the assumption is most appropriate when obstacles are close to each other with deep pockets. The density ratio of the unburnt to burnt gases characterizes the expansion of the combusted gas.

This obstacles-based acceleration is very powerful; it even gets stronger with the increase in the blockage ratio and the thermal expansion factor. To some extent, this kind of acceleration resembles finger-shaped flame acceleration [7, 16], but only it lasts longer. The reason for this is because the pockets filled with a fresh fuel mixture separate the free part of the channel from the 
wall, thereby making it lasting much longer. In this light, the toothbrush mechanism can be treated as unlimited in time, provided the assumptions employed are justified.

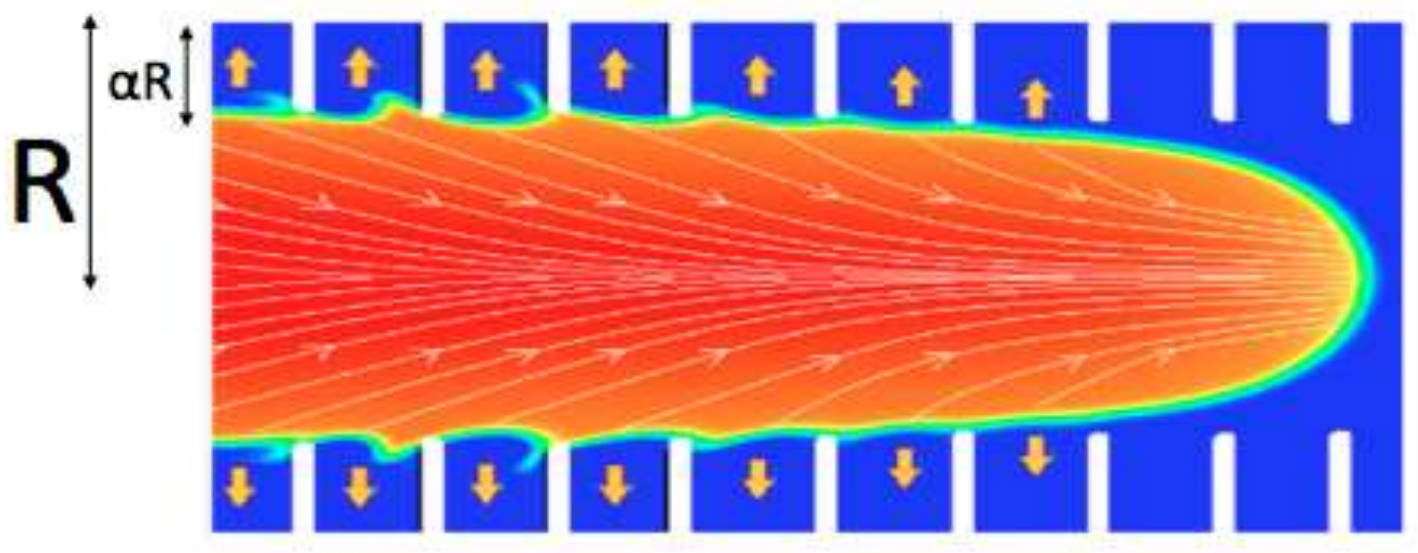

Figure 2.2 Numerical simulations of the toothbrush set of obstacles (snapshot) [17].

A toothbrush mechanism as shown in Figs. 2.1, 2.2 [17] is one of the best configuration of obstacles to use in the semi-open flame explanation. A shockless laminar and inviscid mechanism of high-speed acceleration is found in this demonstration. One end of the pipe is closed, and the flame is ignited at that end, and propagates towards the open one. There is a powerful jet flow along the pipe centerline; it is generated by the cumulative effect of delayed combustion of the pockets between the obstacles and the finger flame mechanism. In this experiment, the turbulent only plays the supplementary role because the flow is so strong that the mechanism is contemptuously laminar.

The flame propagates in 2D semi-open obstructed channels with a half-width $R$ as shown in Figs. 2.1, 2.2 [17]. The rest of the channel is blocked by the obstacles characterized by a blockage ratio $\alpha$ and the spacing between the obstacles $\Delta \mathrm{z}$.

The flame dynamics depends on the size of the obstacles in the semi-open obstructed channel. The bigger the obstacle size, the higher flame acceleration is. When the obstacles' size is smaller than a certain value, flame acceleration is not effectively reduced. The blockage ratio $\alpha$ characterizes the flame shape, velocity, propagation manner and the acceleration rate and it identifies the areas where delayed burning occurs. Additionally, when $\alpha$ is small the flame acceleration halts when it touches the wall. The obstacles should also be thin as to allow them to be numerically modeled as adiabatic surfaces. 
The effect of width of the channel in semi-open obstructed channel has relatively minor effect. Increase in width has a very slight effect in reduction of flame acceleration. Due to the obstacles in the channel the width of the channel is considered as half width. The width of the semi-open obstructed channel does not affect the acceleration mechanism since comb-shaped obstacles allows continuous acceleration even after the flame skirts come into contact with the wall.

There is an increase in the acceleration rate which is caused by delayed combustion in the spaces between the obstacles. This mechanism is scale-invariant and is Reynolds independent. Too large or small spacing between obstacles would lead to lesser explosion severity. The distance between two neighboring obstacles determines the flame dynamic due to the burning rate, while a volume occupied by the obstacles does not participate in flame acceleration. Small obstacles with small distances between them reduce the flame velocity. According to the Bychkov theory, the spacing between the obstacles was used to be small so it would not influence the boundary condition.

The computational platform employed in this thesis uses the following boundary condition:

- Adiabatic Walls/Obstacles. $(\mathbf{n} \cdot \nabla T=0)$

- Isothermal Walls/Obstacles. $\left(T_{w}=\frac{T_{\text {in }}+T_{\text {out }}}{2}\right)$

- Slip Walls/Obstacles. $(\mathbf{n} \cdot \mathbf{u}=0)$

- No-slip Walls/Obstacles. $(\mathbf{u}=0)$

- Right (Open) end: $\rho=\rho_{f}, P=P_{f}, u_{z}=0$

- Left (Closed) end.

- Hemispherical flame (Figure 2.3) ignited from the left (closed) end.

where $\mathbf{n}$ is a normal vector at a surface and $T_{w}$ is the average value at mid-point from both surface boundaries. 


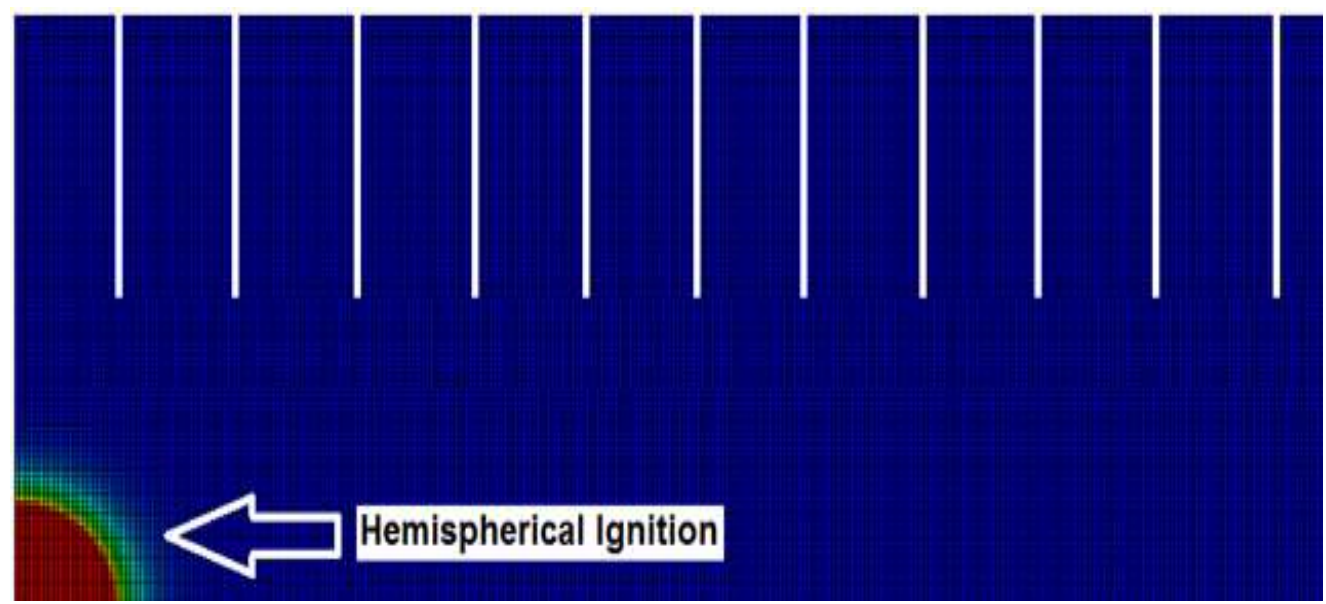

Figure 2.3 A hemispherical flame initiated from the closed end [17].

\subsection{Flame Oscillation/Acceleration in Fully Open Obstructed Channels}

According to some computational analysis, the propagating flame through a narrow pipe is associated with certain behaviors such as different rates of acceleration, oscillations as well as a series of these trends with shocks and turbulence [18].

There is different reactions when the premixed flame was propagated in open-open obstructed and non-obstructed pipes. These behaviors were linked to the mechanistic margin situations, for instance, non-slip walls in pipes with unobstructed walls. Notably, the flame front pushes the whole gas volume generated to the only open side of semi-open channel. Experiments conducted using open-open channels, that is a situation where both ends of the channel are open, provided a different outcome as it was in semi-open obstructed channels. This is because the flame generated gas volume is distributed between the flows towards both open ends while in the semi open pipe, the entire flow pushes towards the single exit. It was observed that the initial gas volume produced was spread between the downstream and upstream flows to the two open ends that reduced the effect on the rate of acceleration and oscillations, subsequently, causing the flame to revolve about the quasi-steady circulation rather than be accelerating.

Notably, speeding up flames in open-open pipes with non-slip adiabatic walls is comparatively moderate. However, the acceleration rate reduces with the reduction in a channel radius. This is conceptually different from the case of obstructed channels. Specifically, they showed that flame acceleration in semi-open pipes with tightly-parked obstacles exhibiting the shape of comb array, 
is a shockless and generally laminar mechanism [18]. In the case of open-open obstructed pipes, acceleration is propelled by delayed combustion occurring in pockets positioned between the two open ends. This effect is equitably strong for broader and active channels where turbulence takes the additional duty. Nevertheless, laboratory and industrial applications on obstructed channels where ignition occurs at one of the open ends introduced the necessity to survey the propagation of premixed flame in open blocked channels. These applications are conducted using twodimension detailed simulations of combustion equations with fully-compressible Arrhenius chemical and hydrodynamics kinetics [18].

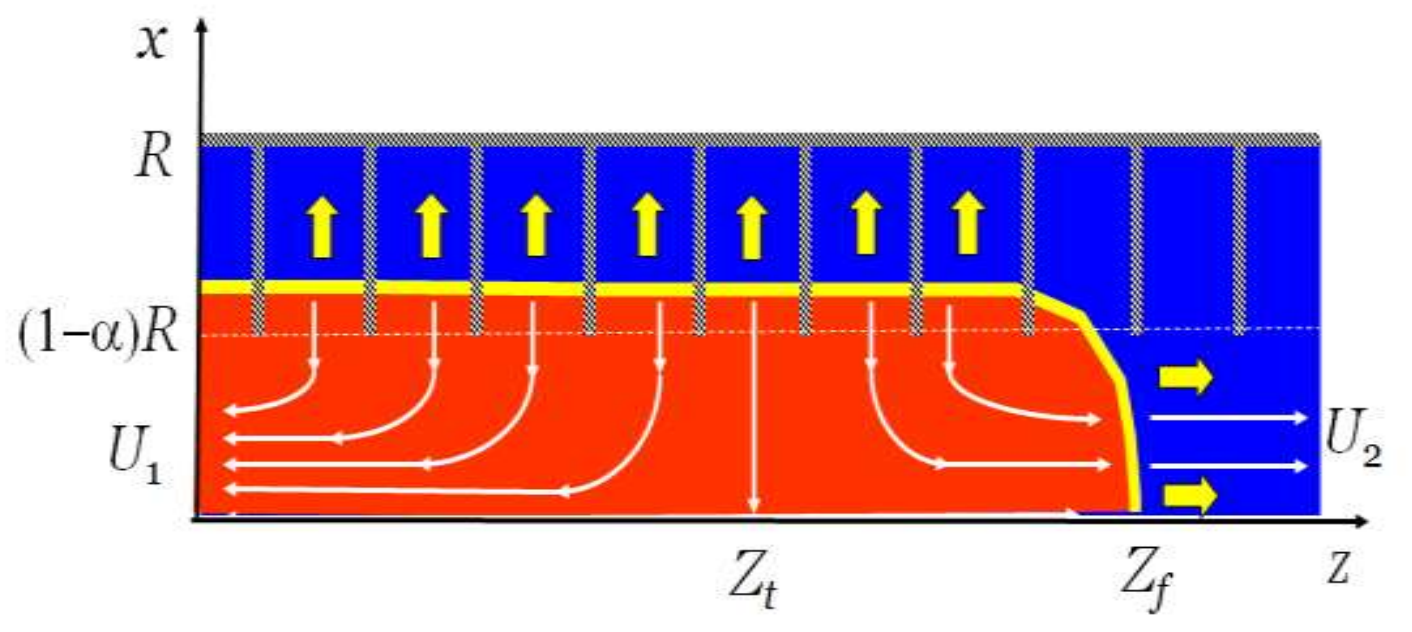

Figure 2.4 Schematic of an open-open obstructed channel [18].

The conceptual variation existing between the open and semi-open pipes is that in semi-open pipes, the additional gas volume generated by delayed burning in the pipes is spread at a particular turning position between the two flows denoted by $\mathrm{Z}_{\mathrm{t}}$. The hot gas flow in Fig. 2.4 is marked by the subscript 1 . As it escapes through the pipe entrance, the turning point is equal to zero while the speed $U_{2}$ and the mixture of the fuel marked with subscript 2, leaving through the tube exit where $\mathrm{Z}=1$ and the rate of burning gas $\mathrm{U}_{2}$. Here $\mathrm{L}$ denotes the total length of the pipe. The amount of $\mathrm{U}_{1}, \mathrm{U}_{2}$, and $\mathrm{Z}_{\mathrm{t}}$ are unknown values, and they have to be calculated from the motion conversation [18].

Clearly, the premixed flame is directed towards a comb-shaped range of obstacles within the inbuilt two-dimensional tube of radius $\mathrm{R}$ in the both ends. This configuration is investigated through solving the burning and hydrodynamic equations together with Arrhenius chemical 
kinetics. A channel with a range of $R / L f \leq 12$ where $L f$ denotes the thickness of the thermal flame. The radius is considered in respect to some ratios of the blockage, $\alpha=1 / 3,1 / 2,2 / 3$, for every $R$.

The boundary conditions for fully open channels:

- Adiabatic Walls/Obstacles. $(\mathbf{n} \cdot \nabla T=0)$

- Slip Walls/Obstacles. $(\mathbf{n} \cdot \mathbf{u}=0)$

- Right (Unburnt) end: $T=T_{f}, \rho=\rho_{f}, U_{z}=-S_{L}$

- Left (Burnt) end: $T=\Theta T_{f}, \rho=\rho_{f} / \Theta, U_{z}=-\Theta S_{L}$

- Planar flame ignited from the left (Burnt) end.

where $\mathbf{n}$ is a normal vector at a surface. 


\section{Chapter 3: Numerical Method}

We perform computational simulations of the hydrodynamic and combustion equations including transport processes and Arrhenius kinetics [19, 20, 21]. Both 2D planar and axisymmetric cylindrical flows will be investigated. In the general form, the governing equations read:

Continuity Equation:

$$
\frac{\partial \rho}{\partial t}+\frac{1}{r^{\beta}} \frac{\partial}{\partial r}\left(r^{\beta} \rho u_{r}\right)+\frac{\partial}{\partial z}\left(\rho u_{z}\right)=0
$$

Navier-Stokes Equations:

$$
\begin{aligned}
& \frac{\partial}{\partial t}\left(\rho u_{r}\right)+\frac{1}{r^{\beta}} \frac{\partial}{\partial r}\left[r^{\beta}\left(\rho u_{r}^{2}-\zeta_{r, r}\right)\right]+\frac{\partial}{\partial z}\left(\rho u_{r} u_{z}-\zeta_{r, z}\right)+\frac{\partial P}{\partial r}+\Psi_{\beta}=0, \\
& \frac{\partial}{\partial t}\left(\rho u_{z}\right)+\frac{1}{r^{\beta}} \frac{\partial}{\partial r}\left[r^{\beta}\left(\rho u_{r} u_{z}-\zeta_{r, z}\right)\right]+\frac{\partial}{\partial z}\left(\rho u_{z}^{2}-\zeta_{z, z}\right)+\frac{\partial P}{\partial z}=0,
\end{aligned}
$$

Energy Equation:

$$
\frac{\partial}{\partial t}+\frac{1}{r} \frac{\partial}{\partial r}\left[r\left((+P) u_{r} \quad{ }_{r, r} u_{r} \quad{ }_{r, z} u_{z}+q_{r}\right)\right]+\frac{\partial}{\partial z}\left[(+P) u_{z} \quad{ }_{z, z} u_{z} \quad{ }_{r, z} u_{r}+q_{z}\right]=0,
$$

Species Equation:

$$
\frac{\partial}{\partial t}(\rho Y)+\frac{1}{r^{\gamma}} \frac{\partial}{\partial r}\left[r^{\gamma}\left(\rho u_{i} Y-\frac{\mu}{\mathrm{Sc}} \frac{\partial Y}{\partial r}\right)\right]+\frac{\partial}{\partial z}\left(\rho u_{z} Y-\frac{\mu}{\mathrm{Sc}} \frac{\partial Y}{\partial z}\right)=-\frac{\rho Y}{\tau_{R}} \exp \left(-E_{a} / R_{p} T\right)
$$

where $\beta=0$ and 1 for 2D and axisymmetric geometries respectively,

$$
\varepsilon=\rho\left(Q Y+C_{V} T\right)+\frac{\rho}{2}\left(u_{z}^{2}+u_{r}^{2}\right)
$$

is the total energy per unit volume, $Y$ the mass fraction of the fuel, $Q$ the energy release from the reaction, and $C_{V}$ the heat capacity at constant volume. The energy diffusion vector $q_{i}$ is given by

$$
\begin{aligned}
& q_{r}=-\mu\left(\frac{C_{P}}{\operatorname{Pr}} \frac{\partial T}{\partial r}+\frac{Q}{\operatorname{Sc}} \frac{\partial Y}{\partial r}\right), \\
& q_{r}=-\mu\left(\frac{C_{P}}{\operatorname{Pr}} \frac{\partial T}{\partial z}+\frac{Q}{\operatorname{Sc}} \frac{\partial Y}{\partial z}\right),
\end{aligned}
$$

The stress tensor $\zeta_{i, j}$ takes the form 


$$
\zeta_{i, j}=\mu\left(\frac{\partial u_{i}}{\partial x_{j}}+\frac{\partial u_{j}}{\partial x_{i}}-\frac{2}{3} \frac{\partial u_{k}}{\partial x_{k}} \delta_{i, j}\right)
$$

in the $2 \mathrm{D}$ configuration $(\beta=0)$, while in the axisymmetric geometry $(\beta=1)$ it reads

$$
\begin{aligned}
& \zeta_{r, r}=\frac{2 \mu}{3}\left(2 \frac{\partial u_{r}}{\partial r}-\frac{\partial u_{z}}{\partial z}-\frac{u_{r}}{r}\right) \\
& \zeta_{z, z}=\frac{2 \mu}{3}\left(2 \frac{\partial u_{z}}{\partial z}-\frac{\partial u_{r}}{\partial r}-\frac{u_{r}}{r}\right) \\
& \zeta_{r, z}=\mu\left(\frac{\partial u_{r}}{\partial z}+\frac{\partial u_{z}}{\partial r}\right)
\end{aligned}
$$

Finally, the last term in Eq. (2) takes the form

$$
\psi_{\beta}=\frac{2 \mu}{3}\left(2 \frac{u_{r}}{r}-\frac{\partial u_{r}}{\partial r}-\frac{\partial u_{z}}{\partial z}\right),
$$

if $\beta=1$, and $\psi_{\beta}=0$ if $\beta=0$. Here $\mu$ is the dynamic viscosity, Pr and Sc are the Prandtl and Schmidt numbers, respectively. We chose $\mu_{f}=1.7 \times 10^{-5} \mathrm{Ns} / \mathrm{m}^{2}$ in the fuel mixture. To suppress the thermal-diffusive instability we assume unity Lewis number $L e \equiv S c / \operatorname{Pr}=1$, with $\operatorname{Pr}=S c=0.75$. The fuel-air mixture and burnt gas are assumed to be a perfect gas with a constant molar mass $m=2.9 \times 10^{-2} \mathrm{~kg} / \mathrm{mol}$, with $C_{V}=5 R_{p} / 2 m, C_{P} \equiv C_{V}+R_{p}=7 R_{p} / 2 m$, and the equation of state

$$
P=\rho R_{p} T / m
$$

where $R_{P} \approx 8.31 \mathrm{~J} /(\mathrm{mol} \cdot \mathrm{K})$ is the universal gas constant. We consider an one-step irreversible Arrhenius reaction of the first order with an activation energy $E_{a}$ and frequency factor corresponding to a characteristic time $\tau_{R}$. In the simulations we took $E / R_{p} T_{f}=32$ in order to have better resolution of the reaction zone. The factor $\tau_{R}$ was adjusted to obtain a particular value of the unstretched laminar flame speed $S_{L}$ by solving the associated eigenvalue problem. The thermal flame thickness is conventionally defined as

$$
L_{f} \equiv \frac{\mu_{f}}{\operatorname{Pr} \rho_{f} S_{L}}
$$


where $\rho_{f}=1.16 \mathrm{~kg} / \mathrm{m}^{3}$ is the unburnt mixture density. We took initial temperature of the fuel mixture $T_{f}=300 \mathrm{~K}$, initial pressure $P_{f}=10^{5} \mathrm{~Pa}$, adiabatic index $\gamma=1.4$, initial expansion ratio $\Theta=8$, and various values of the initial Mach number in the range $10^{-3} \leq M a \equiv S_{L} / c_{s} \leq 10^{-2}$, with the lower limit corresponding to realistic methane and propane flames. By varying the Mach number, we investigated the influence of gas compression on the flame acceleration.

We assumed slip and adiabatic boundary conditions at the tube walls:

$$
\mathbf{n} \cdot \mathbf{u}=0, \quad \mathbf{n} \cdot \nabla T=0,
$$

where $\mathbf{n}$ is the unit normal vector at the walls. At the open tube/channel end, the non-reflecting boundary conditions are applied as initial conditions, we used a hemi-spherical (hemi-circular) flame "ignited" at the channel axis at the closed end of the tube, with its structure given by the analytical solution of Zeldovich and Frank-Kamenetsky (ZFK) [19, 20],

$$
\begin{aligned}
& T=T_{f}+\left(T_{b}-T_{f}\right) \exp \left(-\sqrt{r^{2}+z^{2}} / L_{f}\right), \quad \text { if } \quad z^{2}+x^{2}<r_{f}^{2}, \\
& T=\Theta T_{f}, \quad \text { if } \quad z^{2}+x^{2}>r_{f}^{2}, \\
& Y=\left(T_{b}-T\right) /\left(T_{b}-T_{f}\right), P=P_{f}, u_{x}=0, u_{z}=0 .
\end{aligned}
$$

Here $r_{f}$ is the radius of initial flame ball at the closed end of the tube. The finite initial radius of the flame ball is equivalent to a time shift, which required proper adjustments when comparing the theory and numerical simulations. When necessary, the numerical solution was shifted in time to have the theory and the results are modelled starting from the same point.

A 2D hydrodynamic Navier-Stokes code adapted for parallel computations [21] was used. The numerical scheme is second-order accurate in time and fourth order in space for convective terms, and second order in space for the diffusive terms. The code is robust and accurate; having been successfully used in aero-acoustic applications. The code is available in 2D (Cartesian and cylindrical axisymmetric) and 3D Cartesian versions. In the present work only 2D simulations have been computed to save computational time and to be able to perform a large number of simulation runs required for a thorough investigation of the problem.

A uniform grid with quadratic cells of size $0.2 L_{f}$ was used to ensure isotropic propagation of the curved flame in $\mathrm{x}$ and $\mathrm{y}$ directions. The longitudinal size of the calculation domain changes dynamically, following the leading pressure wave. Splines of the third order were used for re- 
interpolation of the flow variables during periodic grid reconstruction to preserve the secondorder accuracy of the numerical scheme. 


\section{Chapter 4: Results and Discussion}

\subsection{Flame Acceleration in Semi-Open Obstructed Channels}

During propagation, new pockets of fuel mixture are formed in between obstacles, this delayed burning results in the expansion of the gas which then combusts producing a strong jet flow directed towards unobstructed regions. The flow results in the increased acceleration of the flame through the tube, creating new pockets which establish positive feedback with the flame flow thus increasing the acceleration of the flame which detonates after reaching supersonic speeds in relation to the tube walls [15].

The mechanism has a half-width radius $R$ and $\alpha R$ is the length of the obstacle. Also, a blockage ratio represented as $\alpha$. All these parameters have important impact on the flame. Finally, the spacing between the obstacles is $\Delta z$. The $2 \mathrm{D}$ semi-open obstructed channel has one end of the channel closed, and the initial flame structure was imitated by the classical Zeldovich-FrankKamenetsky (ZFK) solution for a hemispherical flame front [17] being ignited at that end of the pipe.

In this section, combination of walls and thermal boundary are considered: a) Slip b) Nonslip c) adiabatic and d) isothermal. These conditions are being studied by computational simulations and these simulations are used to validate if the Bychkov theory \& modeling by employing acceptable assumptions. Simply Bychkov assumptions employ set of simplification such as slip and adiabatic walls/obstacles.

We have simulated and investigated different parameters with varying boundary conditions. Simply we kept two parameters constant and investigated the variation of the other parameter. Figs. 4.1, 4.2 and 4.3, shows a compared three cases of the slip and no slip boundary condition for various $R, \Delta Z$, and . 


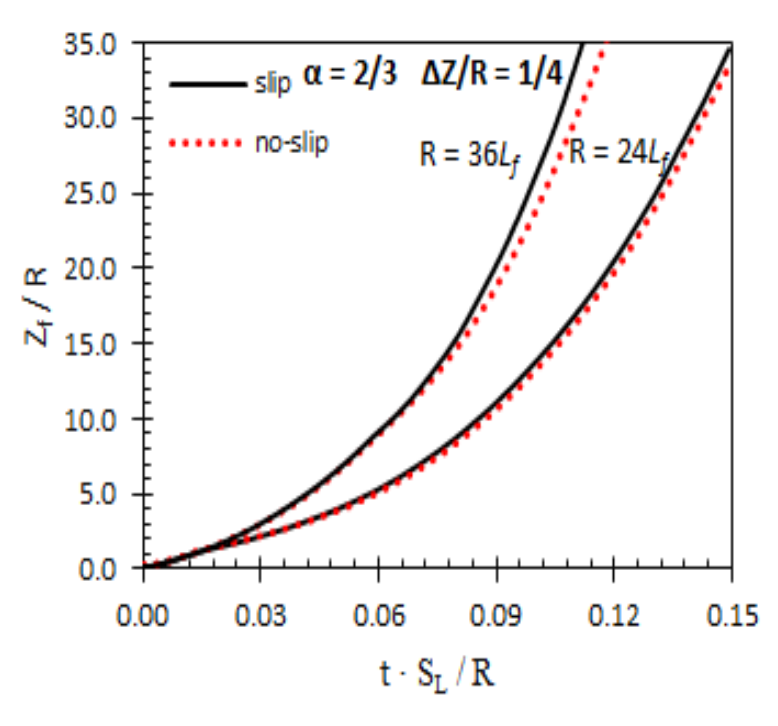

Figure 4.1 The scaled flame position $Z_{f} / R$ vs the scaled time $\tau$ for $R=24 L_{f}, \alpha=1 / 3$ and $\Delta Z / R=1 / 4$.

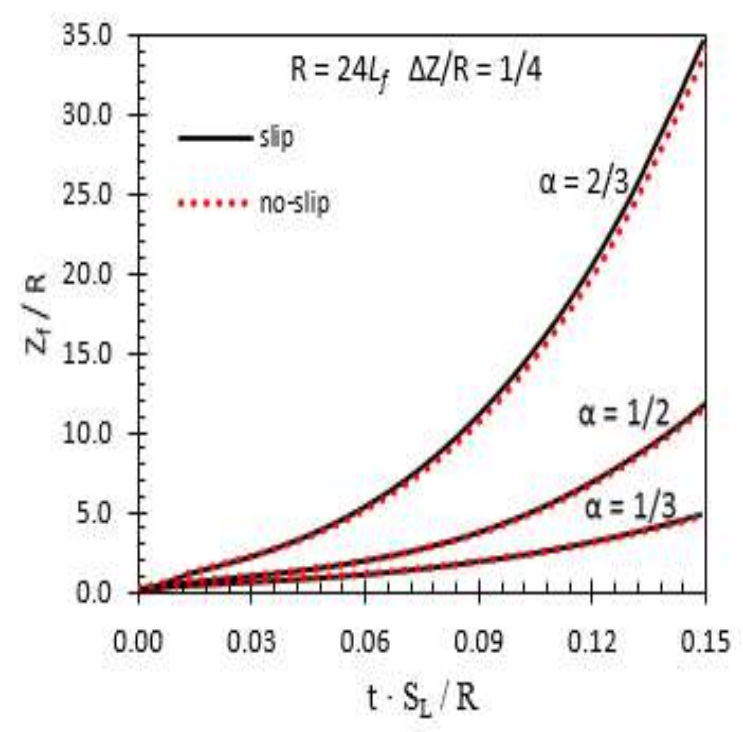

Figure 4.2 The scaled flame position $Z_{f} / R$ vs the scaled time $\tau$ for $R=24 L_{f}, \alpha=1 / 3$, $1 / 2,2 / 3$ and $\triangle Z / R=1 / 4$.

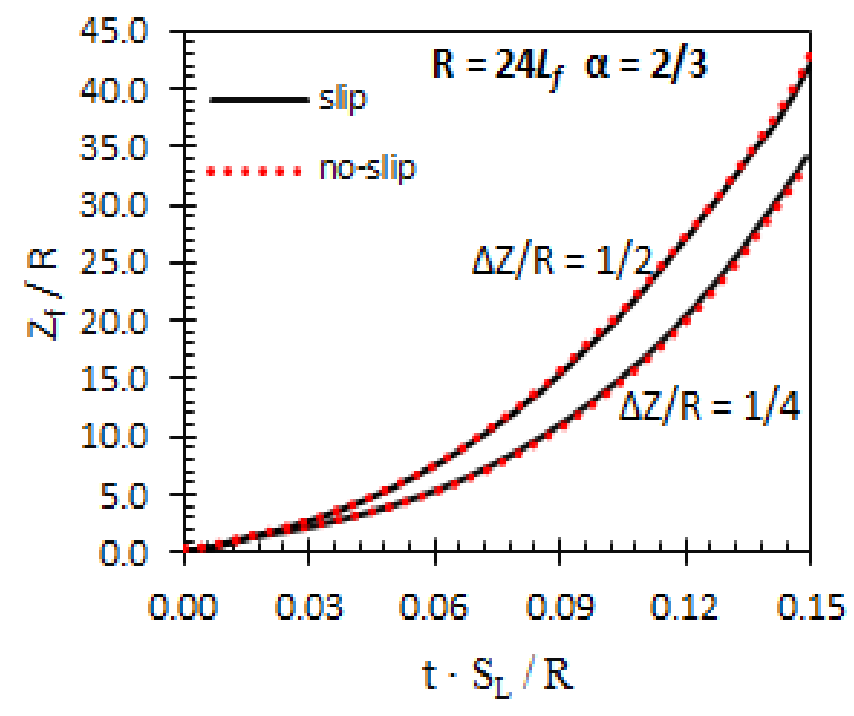

Figure 4.3 The scaled flame position $Z_{f} / R$ vs the scaled time $\tau$ for $R=24 L_{f}, \alpha=2 / 3$ and $\Delta Z / R=1 / 2,1 / 4$.

Figures 4.1, 4.2 and 4.3 above shows the scaled flame tip position $Z_{f} / R$ versus the scaled time $\tau=t S_{L} / R$ for the slip and non-slip boundary conditions. The black solid line is a representation 
of the slip wall condition where the red dash line represents the non-slip wall boundary condition. The effect can barely be noticeable, in fact it is very minor that the walls/obstacles effect when having small obstacles spacing for all given range of the blockage ratio $1 / 3 \leq \alpha \leq 2 / 3$ can be neglected. Moreover, friction slightly moderates flame acceleration for $\Delta Z=R / 4$, and slightly promotes it for $\Delta Z=R / 2$.

Byckhov theory \& modeling employs the freely slip and adiabatic walls/obstacles. Since the impact of the mechanistic boundary condition is minor the Byckhov theory \& modeling of flame acceleration in obstructed pipes is validated. This result is driven by axial direction such that the small obstacle spacing mitigates a potential effect of wall fiction. Additionally, Fig. 4.4, a colored snapshot for $\Delta Z=R / 2$ supports what was concluded.

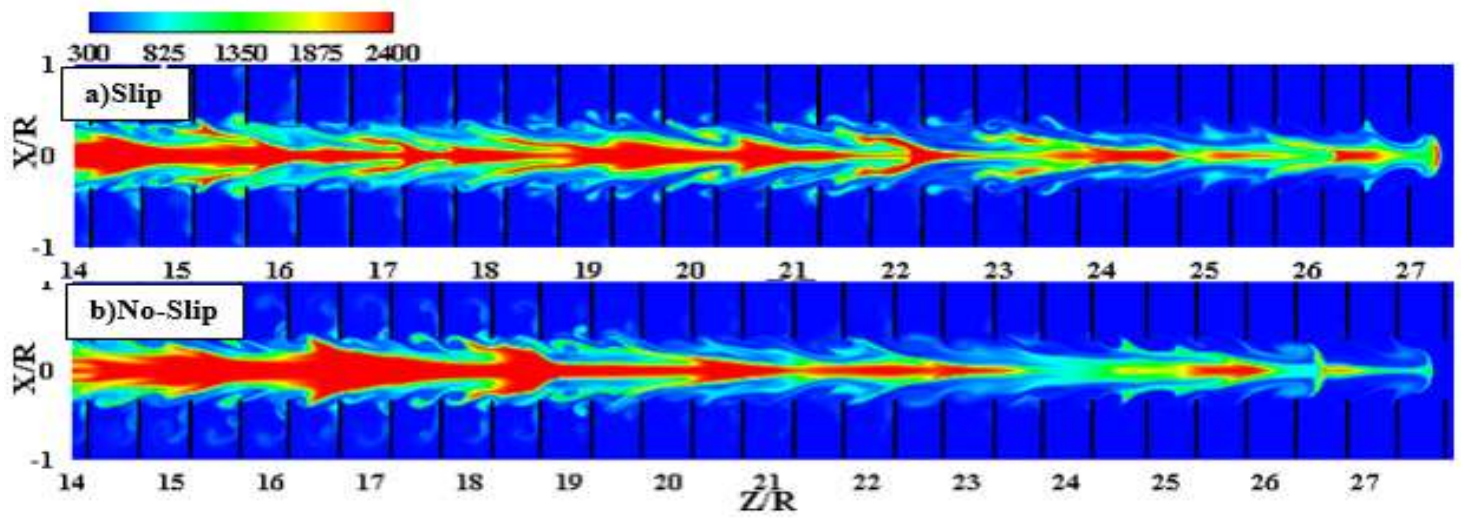

Figure 4.4 The flame evolution in tube of $R=24 L_{f}, \alpha=2 / 3$ and $\Delta Z / R=1 / 2$ : a) slip and b) noslip.

In this study, the aim was to investigate what would happen when the obstacle spacing is increased. In Figs. 4.5, 4.6 and 4.7 we can notice that the results for both slip and non-slip almost coincide for small obstacle spacing when $Z \leq R / 2$ as discussed previously, but the difference between them is observed when $\Delta Z=R$. Additionally, for the slip and non-slip boundary conditions, colored screenshots were taken approximately at the same time from the simulations and are presented in Figs. 4.8, 4.9, and 4.10. It can be seen that there is minor change between the slip and non-slip walls, because the vorticities in the pockets are barely in contact with the walls. 


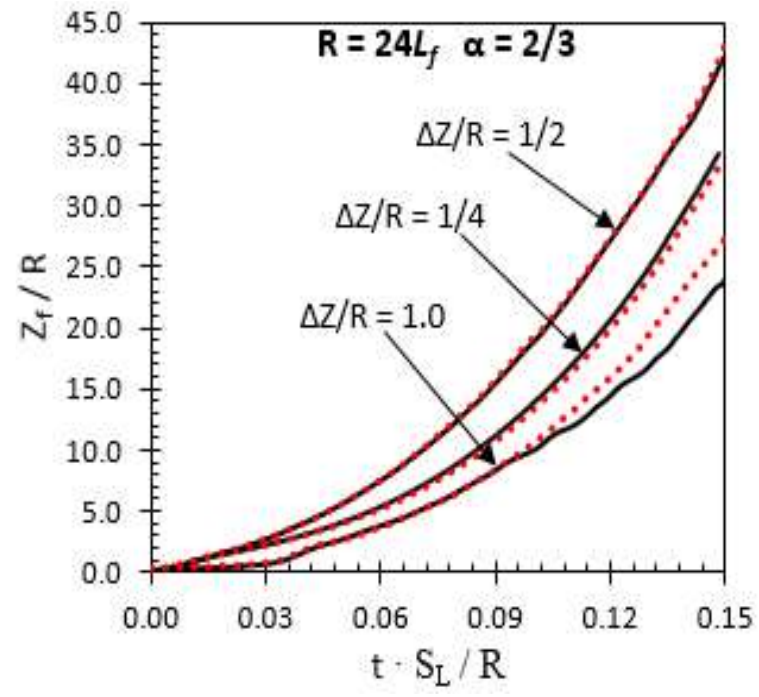

Figure 4.5 The scaled flame position $Z_{f} / R$ vs the scaled time $\tau$ for $R=24 L_{f}, \alpha=2 / 3$ and $\triangle Z / R=1 / 4,1 / 2,1$.

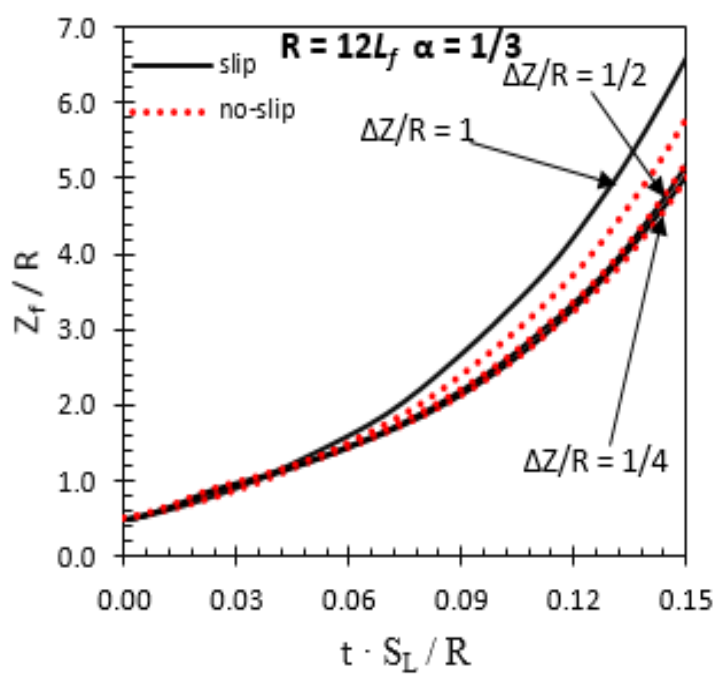

Figure 4.6 The scaled flame position $Z_{f} / R$ $v$ s the scaled time $\tau$ for $R=12 L_{f}, \alpha=1 / 3$ and $\triangle Z / R=1 / 4,1 / 2,1$.

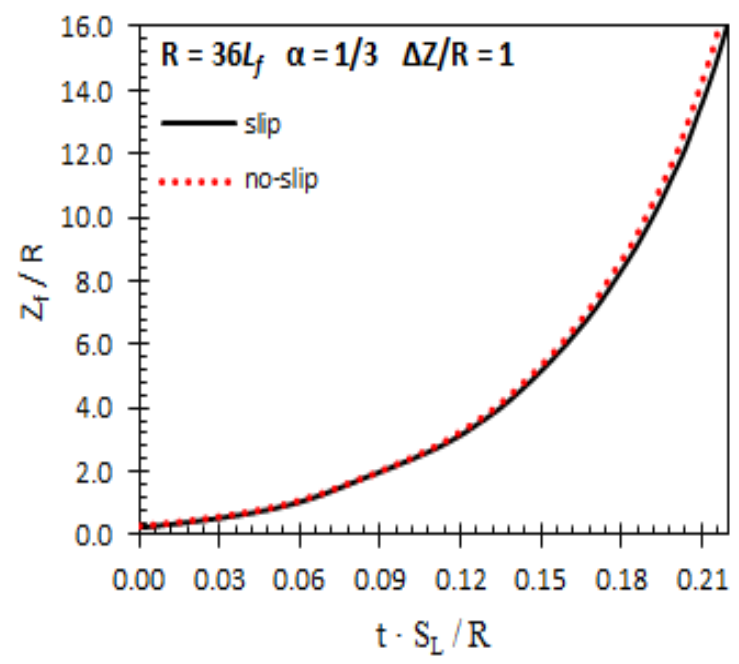

Figure 4.7 The scaled flame position $Z_{f} / R$ vs the scaled time $\tau$ for $R=36 L_{f}, \alpha=1 / 3$ and $\Delta Z / R=1$. 


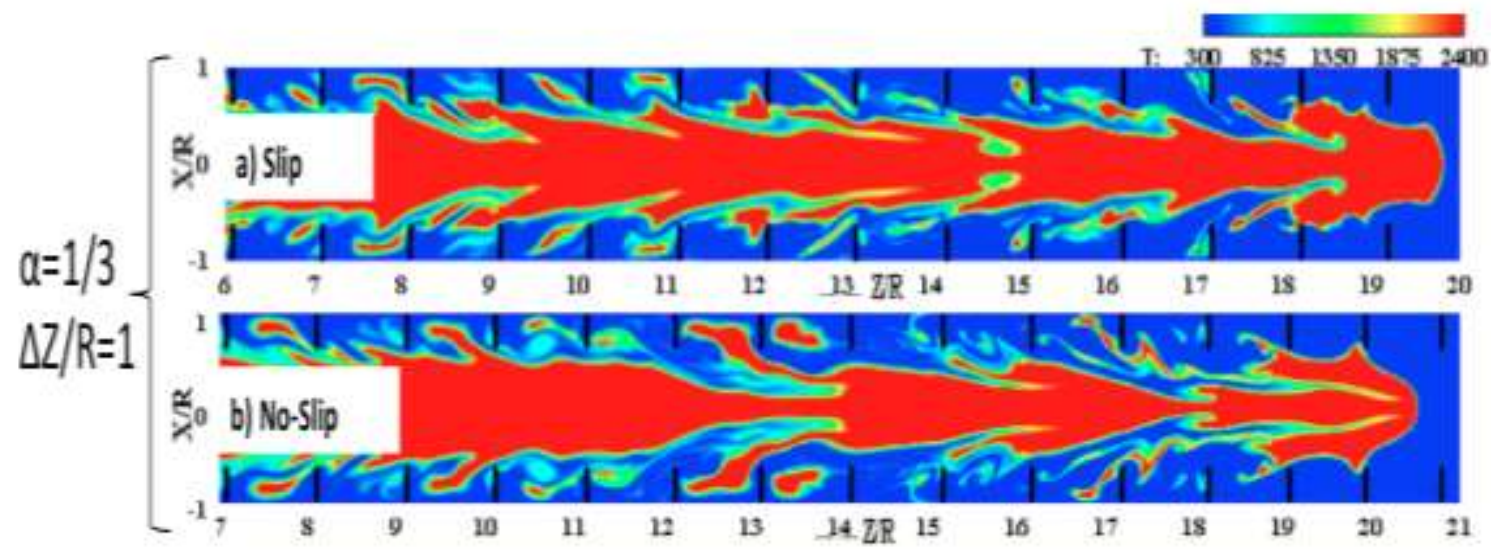

Figure 4.8 The flame evolution in tube of $R=36 L_{f}, \alpha=1 / 3$ and $\Delta Z / R=1:$ a) slip and b) noslip.

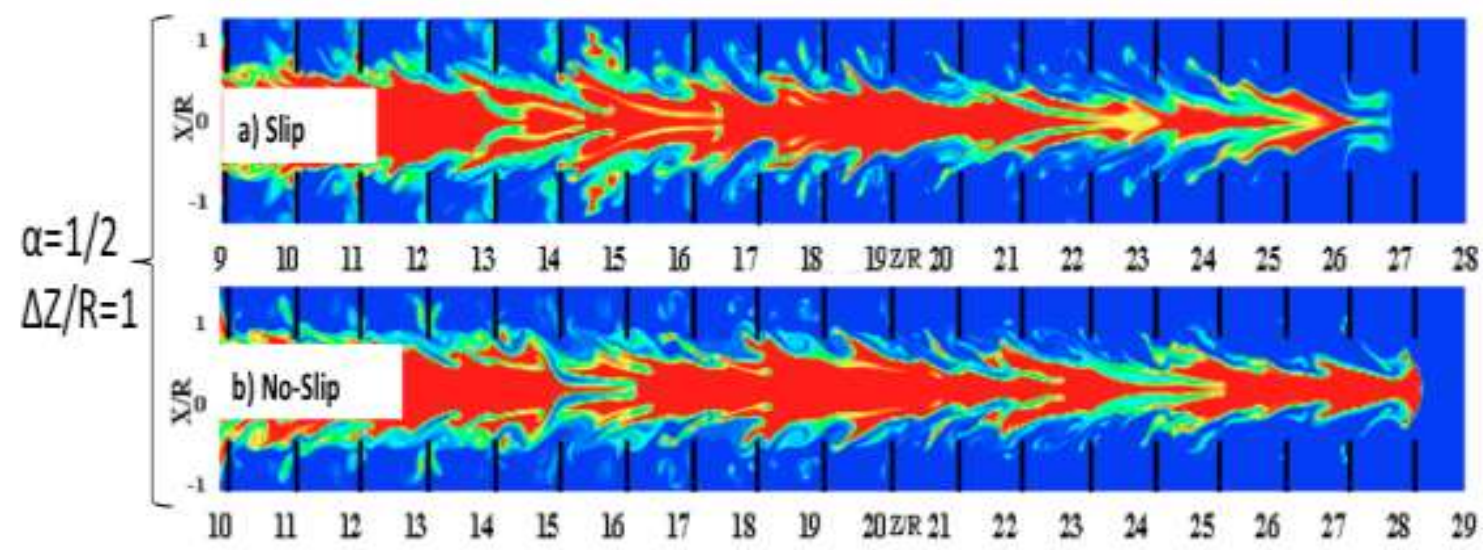

Figure 4.9 The flame evolution in tube of $R=36 L_{f}, \alpha=1 / 2$ and $\Delta Z / R=1$ : a) slip and b) noslip.

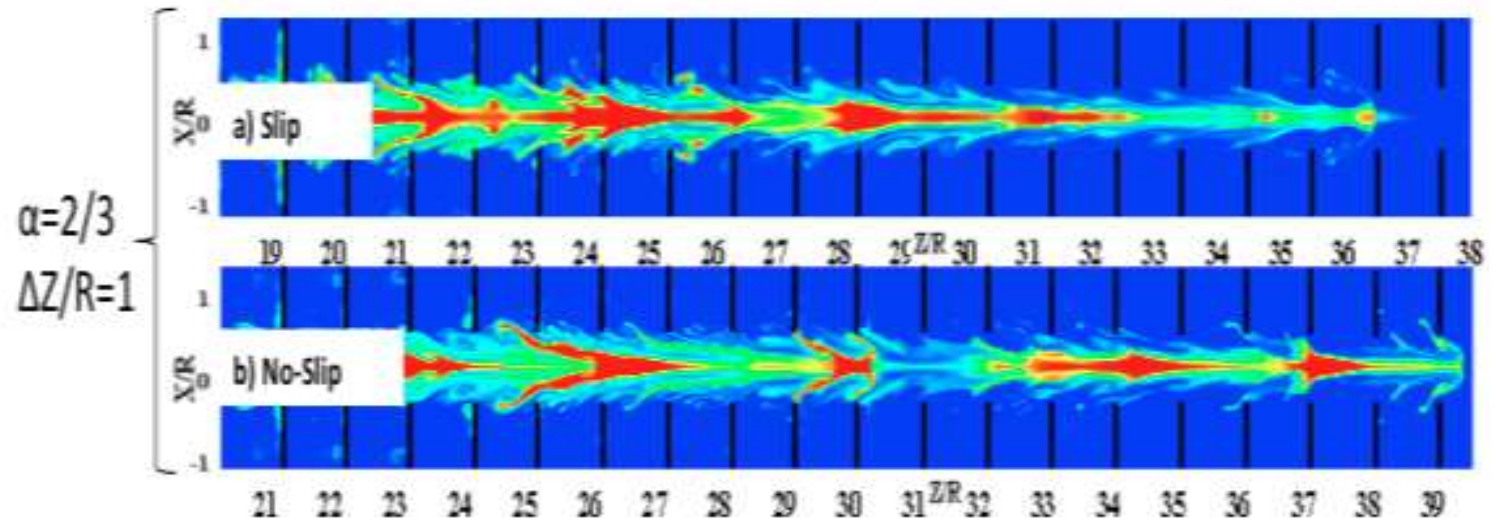

Figure 4.10 The flame evolution in tube of $R=36 L_{f}, \alpha=2 / 3$ and $\Delta Z / R=1:$ a) slip and b) noslip. 
We also studied the case when we have $\Delta Z=2 R$ and a remarkable difference between slip and non-slip was observed as shown in Fig. 4.11. This conclude that for large obstacles spacing the wall friction promotes flame acceleration when comparing the non-slip condition to the slip condition. Moreover, in Fig. 4.12 the vorticity is noticeable and the vorticity progresses differently within slip and non-slip walls. Since Bychkov doesn't consider vorticity it can be concluded that when having large obstacles spacing $\Delta Z<<R$ the wall boundary condition must be considered and this does not align with Bychkov theory \& modeling anymore.

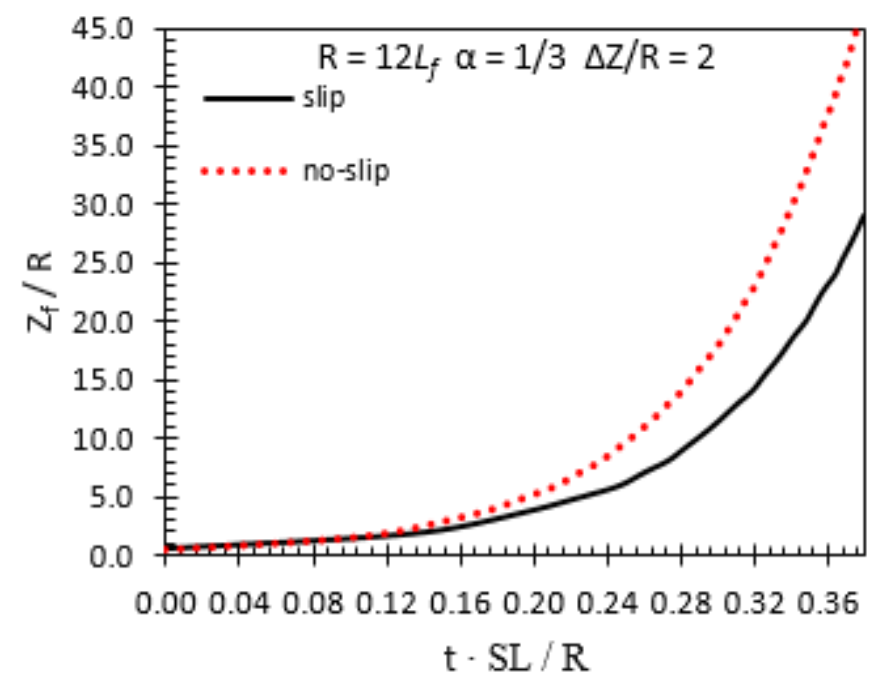

Figure 4.11 The scaled flame position $Z_{f} / R$ vs the scaled time $\tau$ for $R=12 L_{f}, \alpha=1 / 3$ and $\Delta Z / R=2$.
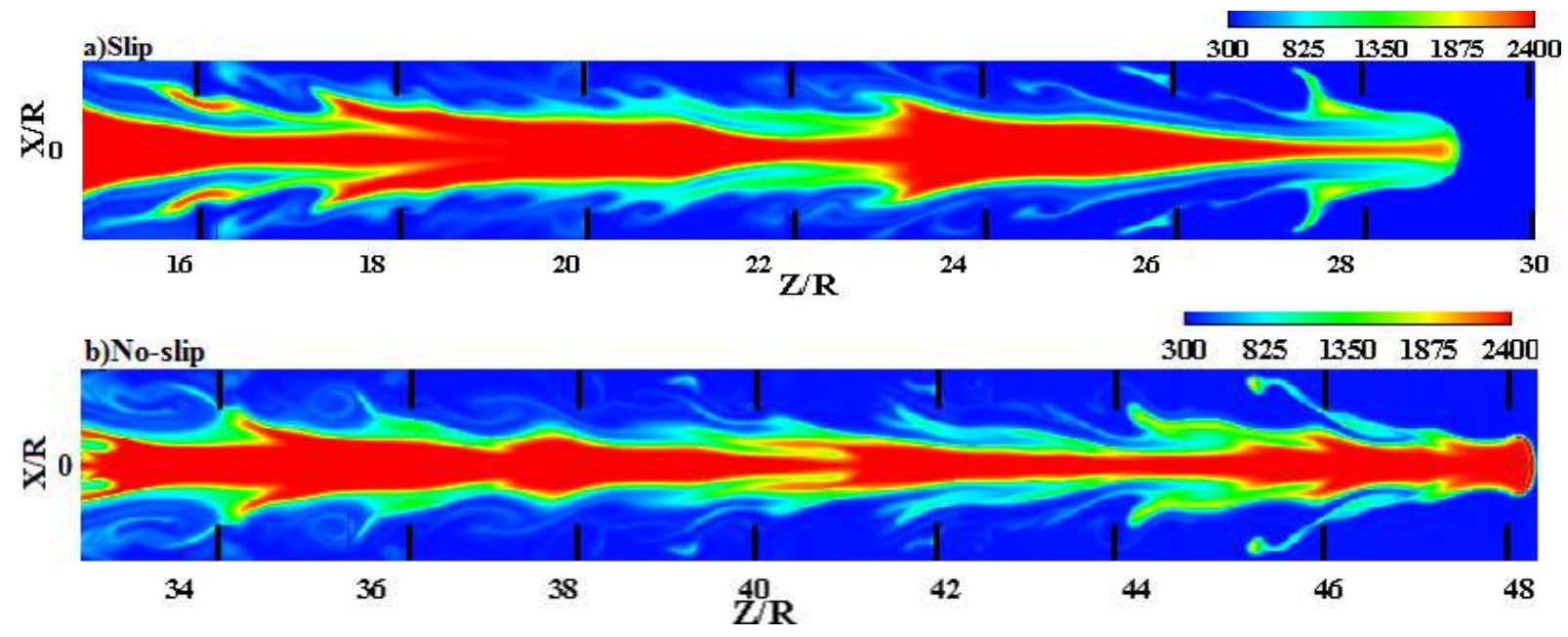

Figure 4.12 The flame evolution in tube of $R=12 L_{f}, \alpha=1 / 3$ and $\Delta Z / R=2:$ a) slip and b) no-slip. 
The second goal was to investigate the effect of thermal boundary condition. The results suggested the same explanation when studying the mechanistic boundary condition (slip and non-slip) at the walls/obstacles in obstructed channels. Fig. 4.13 shows the scaled flame position $U_{t} / S_{L}$ versus the scaled time $\tau=t S_{L} / R$ with black line representing the adiabatic walls/obstacles and blue, green and red lines representing the isothermal walls/obstacles at different room temperatures $298 \mathrm{~K}, 600 \mathrm{~K}$ and $1000 \mathrm{~K}$, respectively. Fig. 4.14 also graphing the scaled flame position $U_{t} / S_{L}$ versus the scaled time $\tau=t S_{L} / R$ at various $\alpha$ and the isothermal and adiabatic boundary conditions shown by the solid and the dashed lines, respectively. Both Figs. 4.13 and 4.14 [17] show minor effect between the adiabatic and isothermal walls/obstacles. Byckhov theory \& modeling employs the adiabatic walls/obstacles and since the effect of the thermal boundary condition is minor here then Byckhov theory \& modeling of flame acceleration in obstructed pipes is validated. The colored snapshots represented in Figs 4.15 and 14.16 [17] are further proof of the minor effect of the thermal boundary condition.

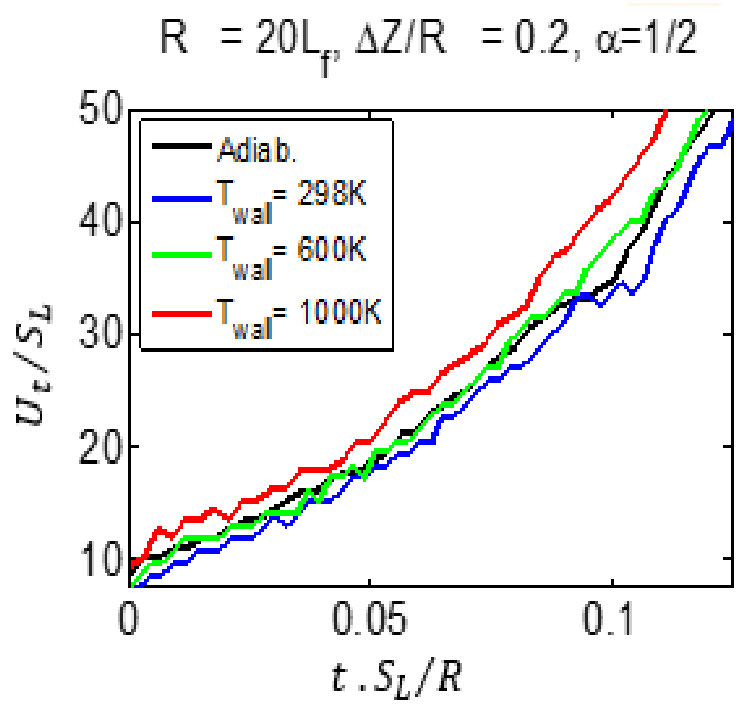

Figure 4.13 The scaled flame position $U_{t} / S_{L} v s$ the scaled time $\tau$ for $R=20 L_{f}, \alpha=1 / 2$ and $\Delta Z / R=$ 0.2. [17]

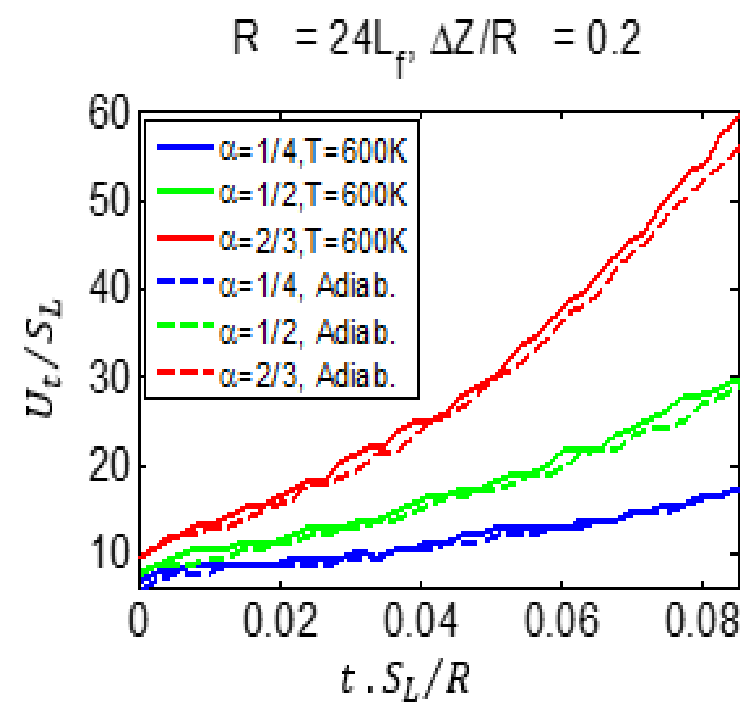

Figure 4.14 The scaled flame position $U_{t} / S_{L} v s$ the scaled time $\tau$ for $R=24 L_{f}$, and $\Delta Z / R=0.2$ at different $\alpha$. [17] 


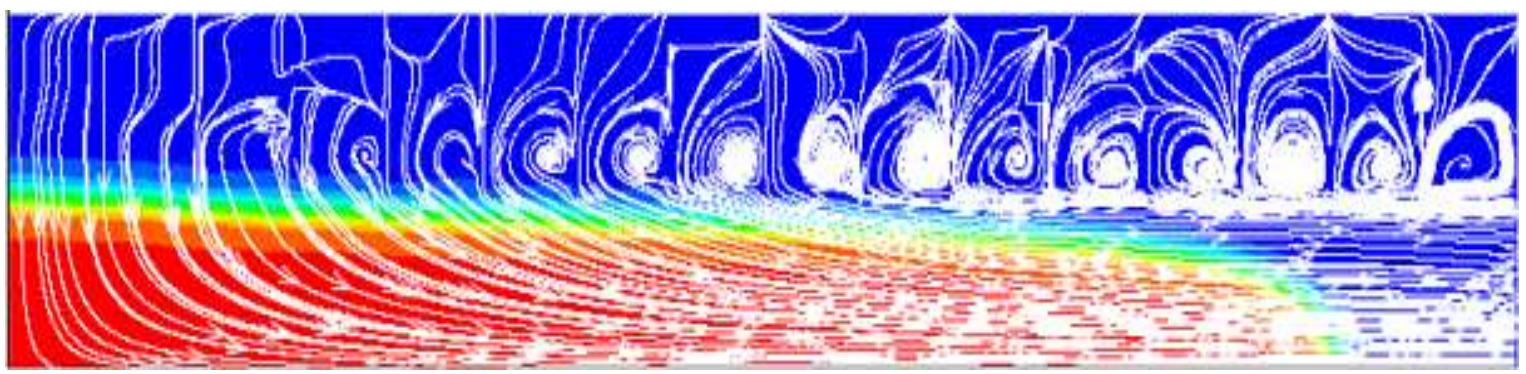

Figure 4.15 The flame evolution in tube of $R=20 L_{f}, \alpha=1 / 2$ and $\Delta Z / R=0.2$ for Adiabatic walls/obstacles. [17]

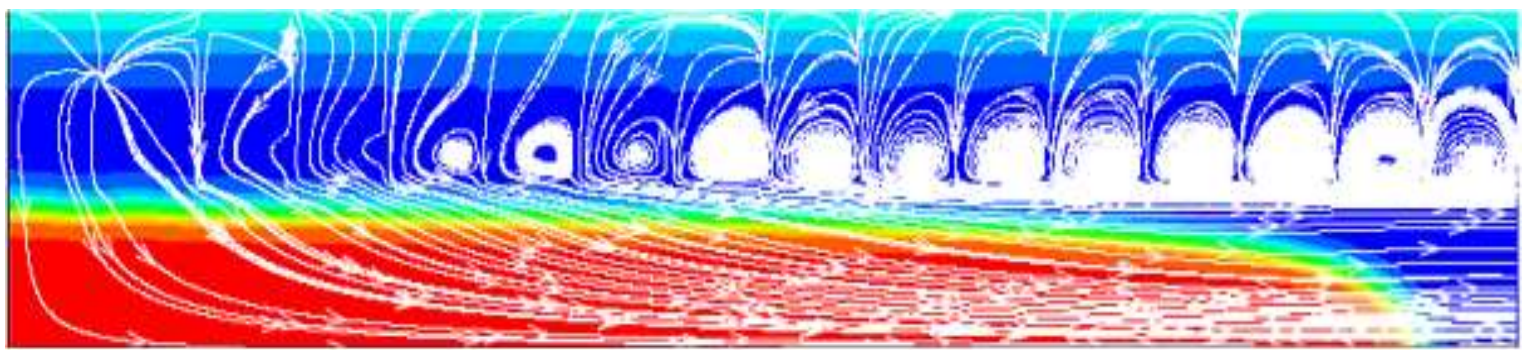

Figure 4.16 The flame evolution in tube of $R=20 \mathrm{Lf}, \alpha=1 / 2$ and $\Delta Z / R=0.2$ for Isothermal (1000K) walls/obstacles. [17]

\subsection{Flame Oscillation/Acceleration in Fully Open Obstructed Channels}

In this section, the current investigations are being studied by computational simulations similar to the previous section. Unambiguously, the premixed flame is directed towards a comb-shaped range of obstacles with an inbuilt two-dimensional tube of radius $\mathrm{R}$ in the both ends. This configuration is investigated through solving the burning and hydrodynamic equations together with Arrhenius chemical kinetics. Channel widths in a range of $R / L_{f} \leq 24$, where $L_{f}$ denotes the thickness of the thermal flame, are considered. The radius is considered in respect to some ratios of the blockage, $\alpha=1 / 3,1 / 2,2 / 3$, for every $R$. We also investigated the impact of the thermal expansion and the spacing between the obstacles. The initial flame structure for the $2 \mathrm{D}$ fully open obstructed channel was imitated by the classical Zeldovich-Frank-Kamenetsky (ZFK) solution for a planar flame front [3] ignited at a distance of $50 L_{f}$ from the left (burnt) end of the pipe. After an extensive analysis of premixed flame propagation and a flame generated velocity field, it was concluded that the length and the curvature of the flame front change with the propagation of the flames. 
Using a scaled burning rate $U_{w} / S_{L}$ the flame dynamics and velocity evolution during the oscillation were investigated. Figs. 4.17, 4.18 and 4.19 below illustrate this evolution:

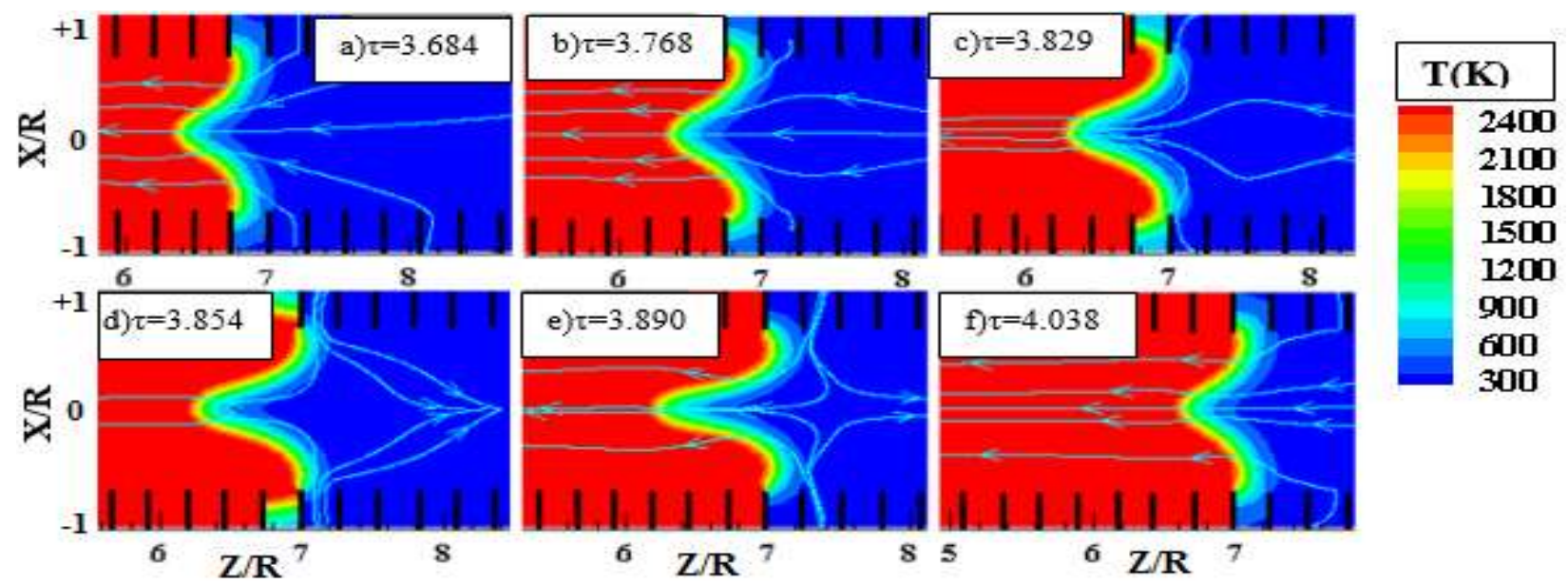

Figure 4.17 Evolution of the flame and the flow in an obstructed channel of $R=12 L_{f}$ and $\alpha=$ $1 / 3$.

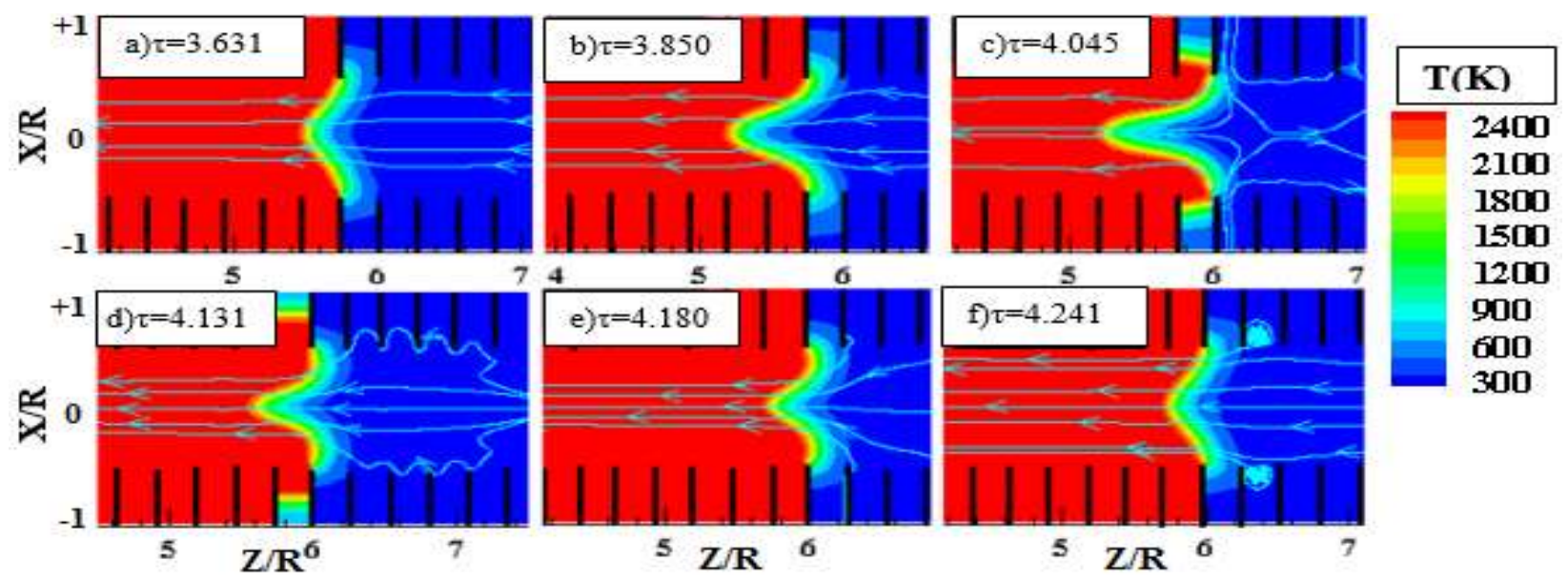

Figure 4.18 Evolution of the flame and the flow in an obstructed channel of $R=12 L_{f}$ and $\alpha=$ $1 / 2$. 


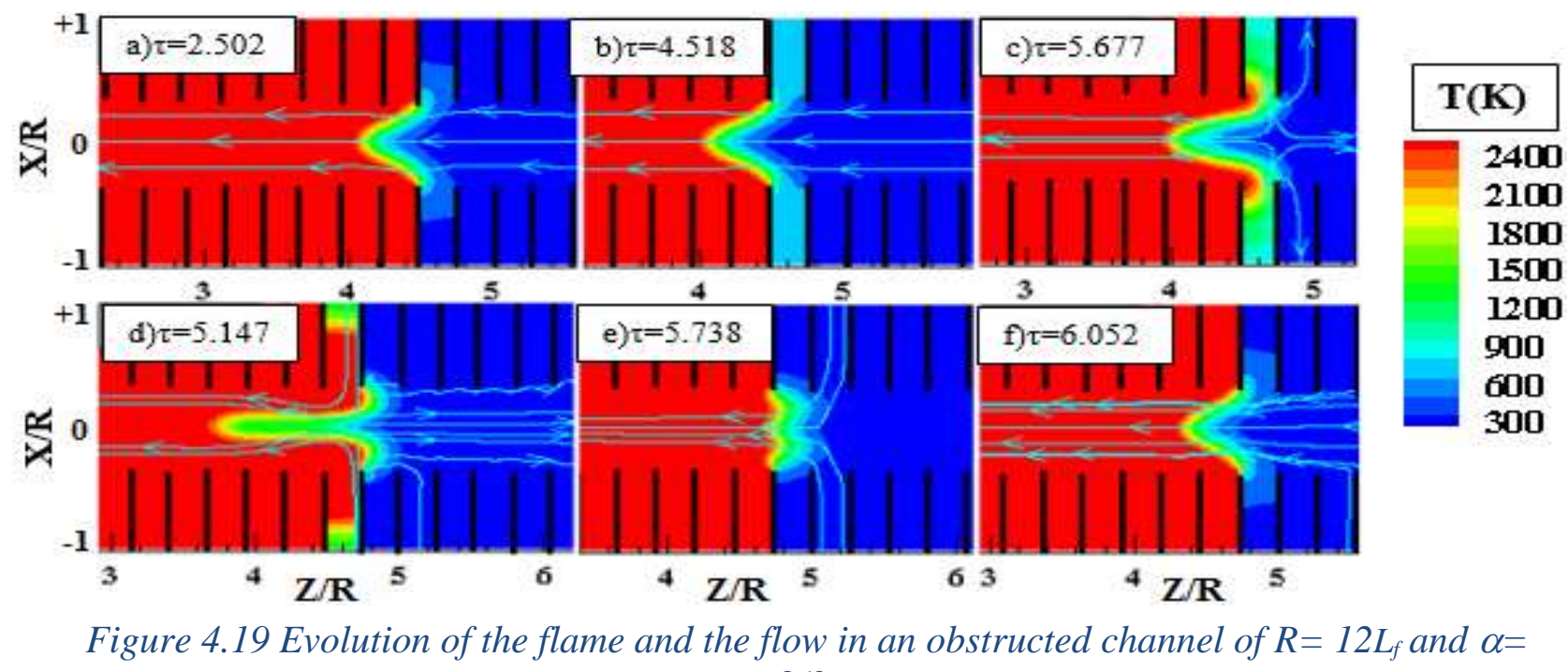
$2 / 3$.

The colors of temperature snapshot represent the flame. A temperature of $300 \mathrm{~K}$ is represented by blue and $2400 \mathrm{~K}$ is red flame. The shapes of the flames differ at different $\alpha$ values. At the free area of the channels the flame acquired a concave shape and directed towards the centerline.

For the first case, the blockage ratio, $\alpha=1 / 3,1 / 2,2 / 3$, for $\operatorname{Re}=12,24$ and $\Delta \mathrm{Z} / \mathrm{R}=0.25$ were investigated. The duration of each stationary stage directly increases with an increase in $\alpha$ as shown by the colored snapshots (a) - (b) in Figs. 4.17, 4.18 and 4.19. While the concavity of the flame front decreases with an increase in $\alpha$. At the initial oscillations stage, the flame consumes the fuel mixture while the burning gas expands. This leads to the generation of a new gas that bends the flame segment hence the convex shape. As a result of the convex shape the surface area of the flame front increases thus enhancing flame acceleration. An increase in the flameout length increases the burning rate enabling the flame to move to the next pocket which causes its front to break and eventually to the termination of the flame. The colored snapshots from (c) to (e) Figs. 4.17, 4.18 and 4.19 illustrate how the flames decelerate due to the breakage of the flame front until they obtain the shapes illustrated by Figures $4.17 \mathrm{f}$ and $4.18 \mathrm{f}$. This constant acceleration and deceleration scenario yields the flame oscillations. 

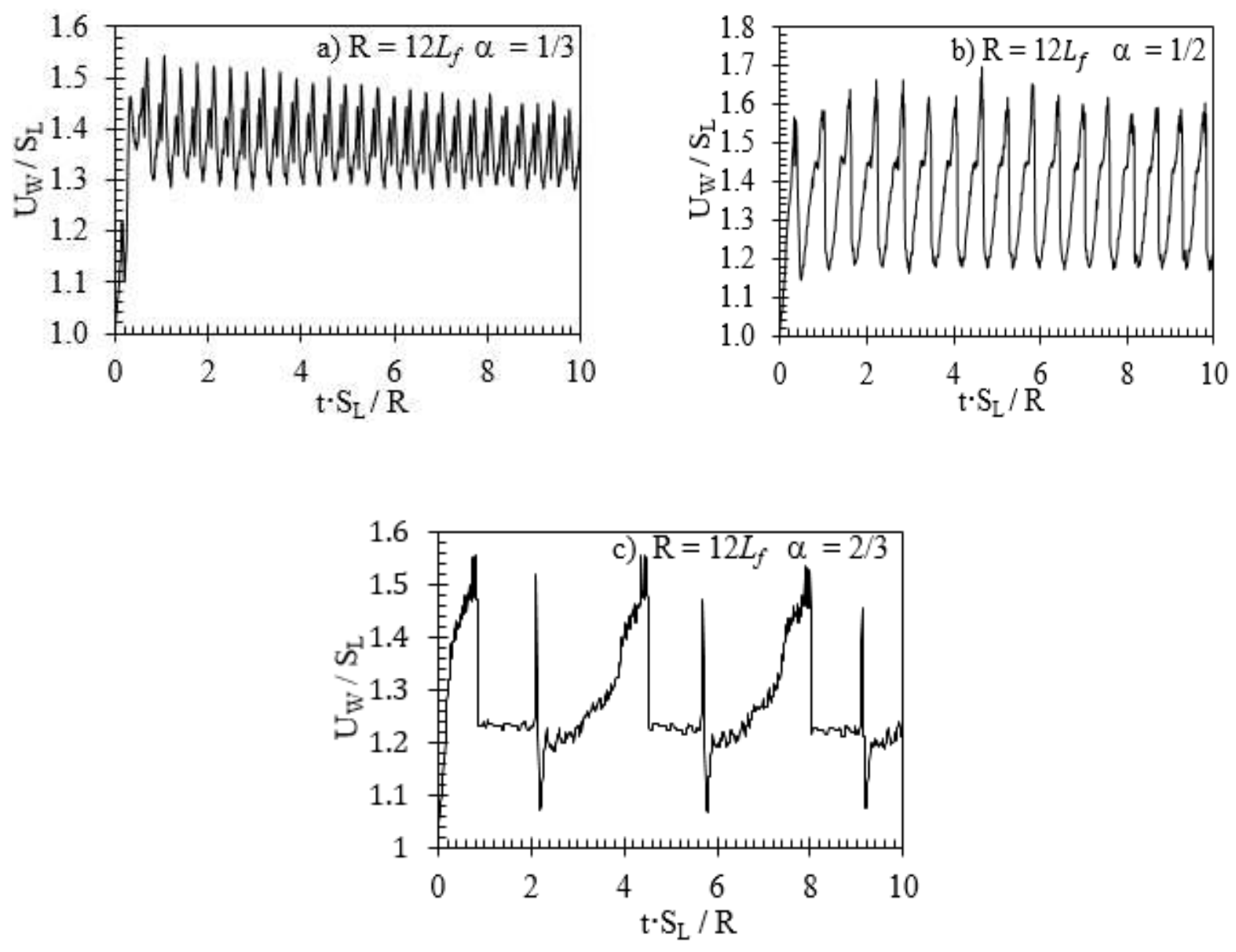

Figure 4.20 The scaled burning rate $U_{t} / S_{L} v$ s the scaled time $\tau$ for $R / L_{f}=12$ and $\alpha=1 / 2,1 / 3$, $2 / 3, \Delta Z / R=1 / 4$.
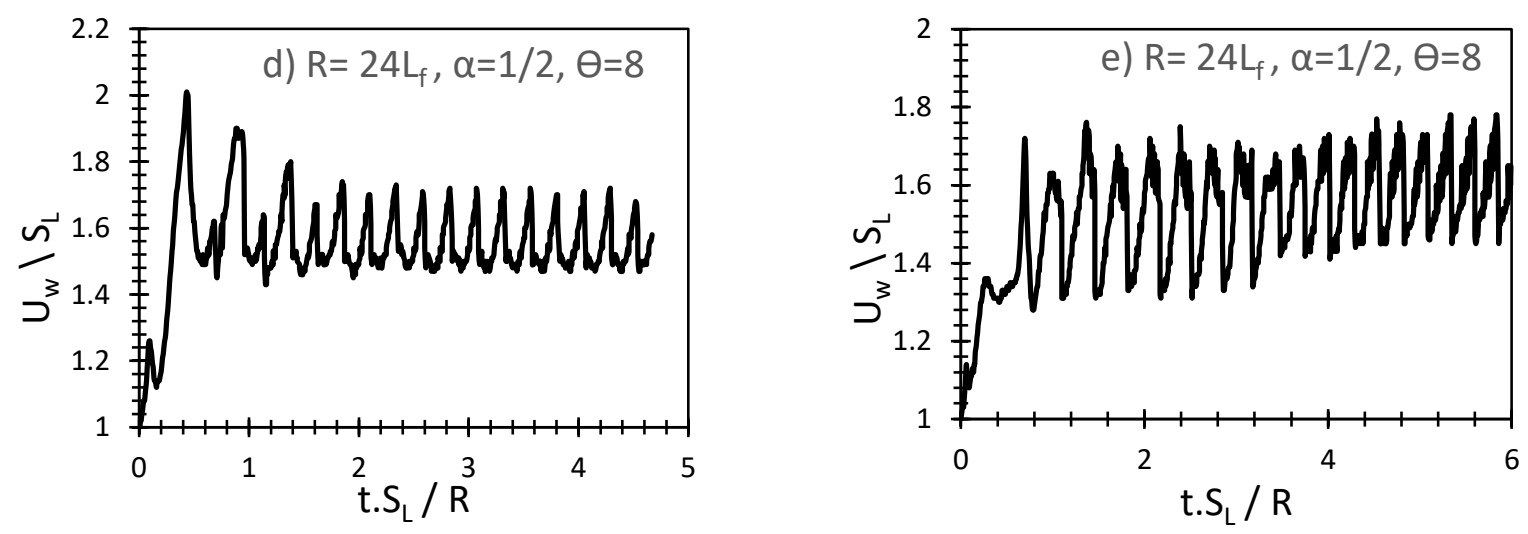

Figure 4.21 The scaled burning rate $U_{t} / S_{L} v$ s the scaled time $\tau$ for $R / L_{f}=24$ and $\alpha=1 / 2,2 / 3$ $\Delta Z / R=1 / 4$. 
Fig. 4.20 is a graph of time evolution against the burning rate of the flame that was plotted to quantify the fame oscillations. Fig. 4.20 (a-c) represents the flame oscillations with $R=12 L_{f}$ and $\alpha=1 / 3,1 / 2,2 / 3$ respectively. The oscillation amplitude gradually reduces with time. The oscillations of the burning rate and non-linearity increase with an increase in $\alpha$ and the period of oscillations increases such that the most profound nonlinearity is achieved at $\alpha=2 / 3$. The direct relationship between nonlinearity and $\alpha$ is a result of ratio between response of the large blockage to the deeper pockets. Moreover, Fig. 4.21d, e describes a twice wide channel, with $R=$ $24 L_{f}$ with the blockage ratio $\alpha=1 / 2,2 / 3$ with $\Theta=8$. Fig. $4.21 \mathrm{~d}$ is in many ways similar to Fig. $4.20 \mathrm{~b}$ despite the difference in their amplitudes and scale oscillation period. The scaled oscillation period in Fig. 4.20b is twice that of Fig. 4.21d. However, Fig 4.20b with $R=12 L_{f}$ and $4.21 \mathrm{~d}$ with $R=24 L_{f}$ have close oscillation periods when considering the dimensional time. The same trend applies to Fig. 4.20c and Fig. 4.21e.

Not only the impact of the blockage ratio, but also the spacing between the obstacles $\Delta \mathrm{Z}$ was investigated. The same finding that was previously discussed for the blockage ratio was observed here. The oscillations of the burning rate and non-linearity increase with an increase in $\Delta \mathrm{Z}$. As seen in Fig. 4.22, when $\Delta \mathrm{Z}$ increases, the period of oscillations increases such that the most profound nonlinearity is achieved at $\Delta \mathrm{Z} / \mathrm{R}=1$.

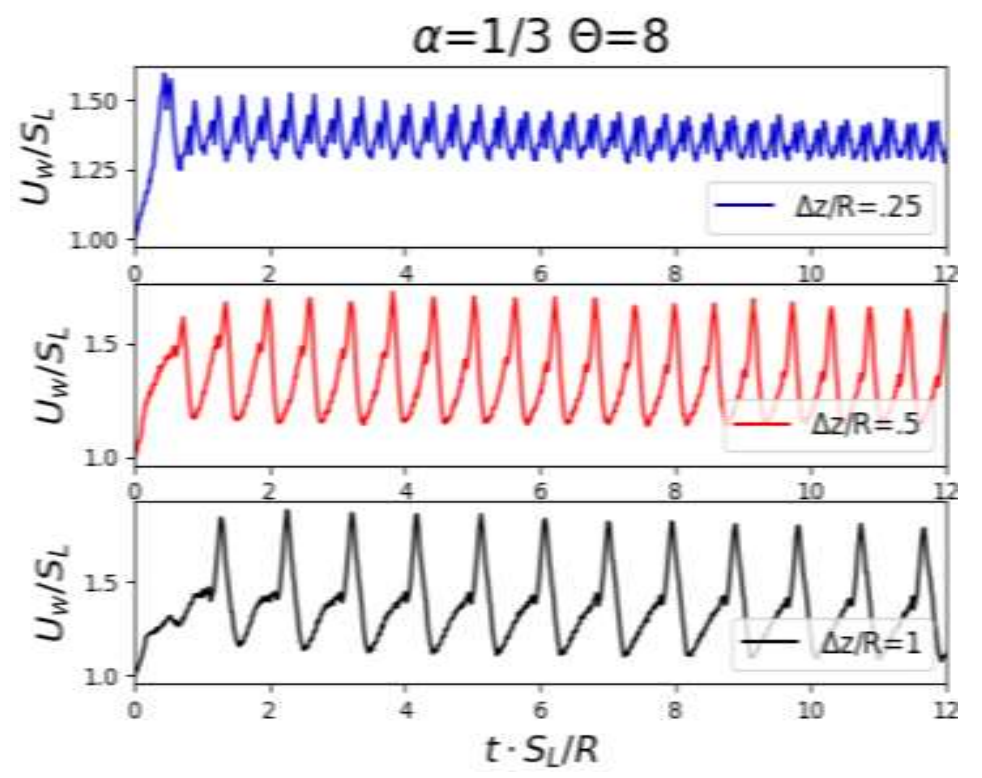

Figure 4.22 The scaled burning rate $U_{w} / S_{L}$ vs the scaled time $t S_{L} / R$ for the thermal expansion ratio $\theta=8$, the blockage ratio $\alpha=1 / 3$ and various obstacle spacing $\Delta Z / R=0.25$, blue; $\Delta Z / R=0.5$, red; and $\Delta Z / R=1$, black. 
Next, the impact of the thermal expansion $\Theta=\rho_{\text {unburnt }} / \rho_{\text {burnt }}$ was evaluated for different blockage ratios which play a key role here as shown in Figs. 4.23, 4.24 and 4.25. For larger $\Theta$ the effect of the blockage ratio becomes weaker on the burning rate and the growing of the oscillation period. Likewise, Figs. 4.26 and 4.27 notice an increase in $\Theta$ leading to decrease of the burning rate and the amplitude. Correspondingly in Fig. 4.28, a reduction in $\Theta$ results in an increase of the period of oscillation.

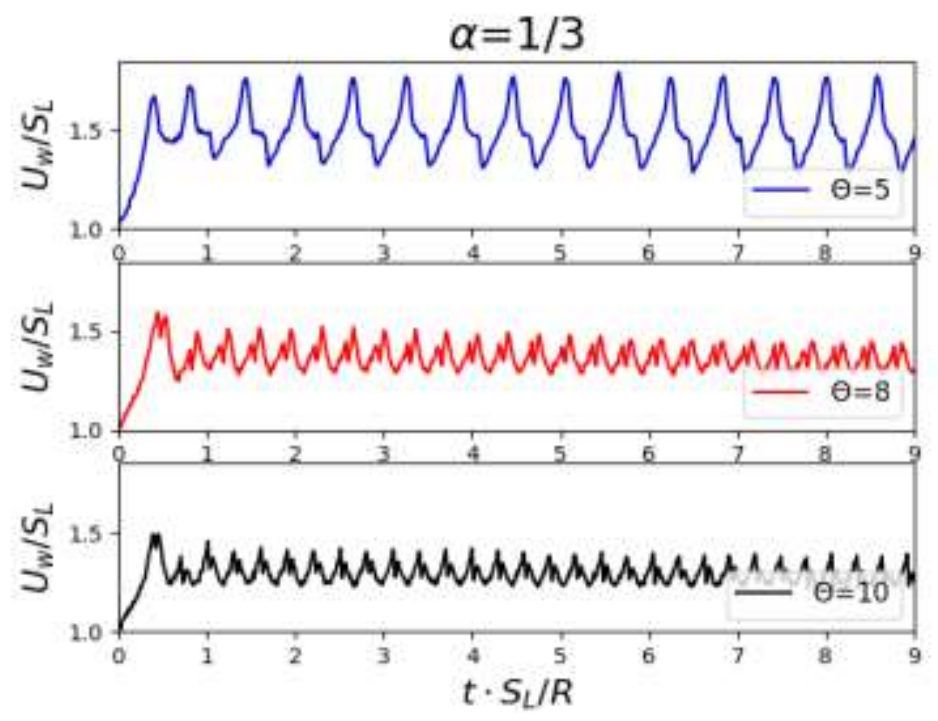

Figure 4.23 The scaled burning rate $U_{w} / S_{L}$ vs the scaled time $t S_{L} / R$ for $\Delta Z / R=0.5$ and blockage ratios $\alpha=1 / 3$; with thermal expansions $\theta=5$, blue; $\theta=8$, red; and $\theta=10$, black.

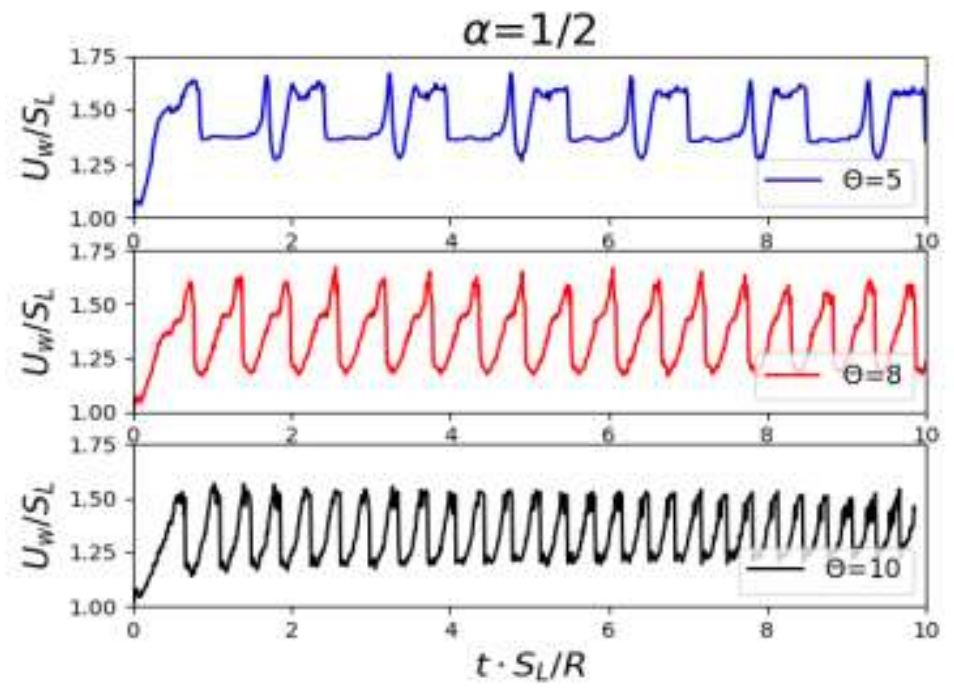

Figure 4.24 The scaled burning rate $U_{w} / S_{L}$ vs the scaled time $t S_{L} / R$ for $\Delta Z / R=0.5$ and blockage ratios $\alpha=1 / 2$; with thermal expansions $\theta=5$, blue; $\theta=8$, red; and $\theta=10$, black. 


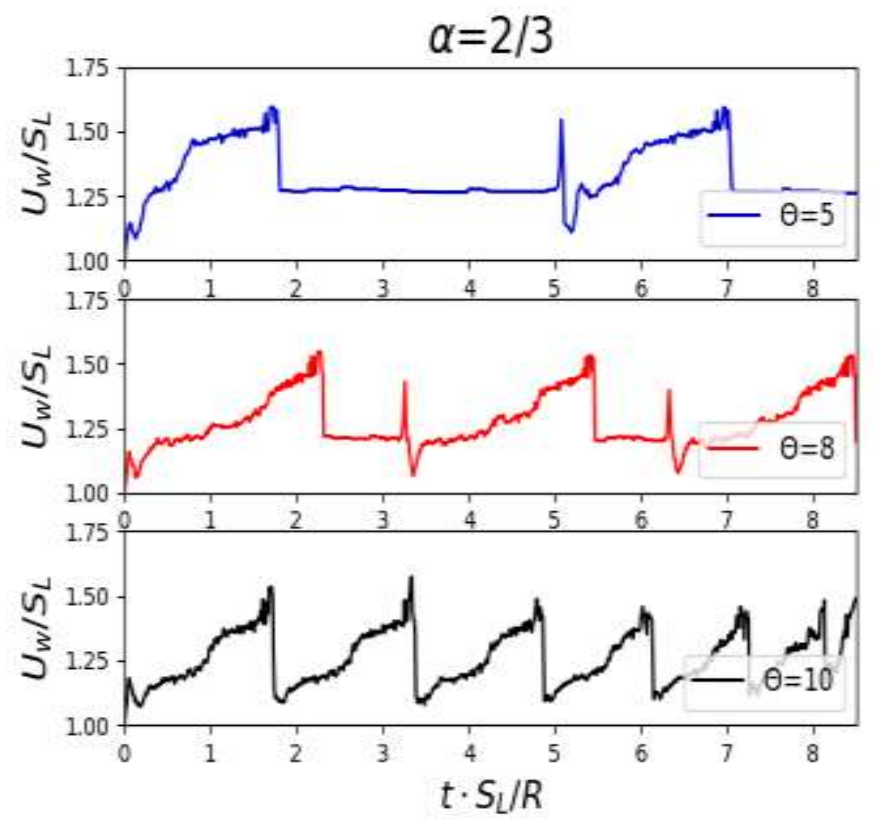

Figure 4.25 The scaled burning rate $U_{w} / S_{L}$ vs the scaled time $t S_{L} / R$ for $\Delta Z / R=0.5$ and blockage ratios $\alpha=2 / 3$; with thermal expansions $\theta=5$, blue; $\theta=8$, red; and $\theta=10$, black.

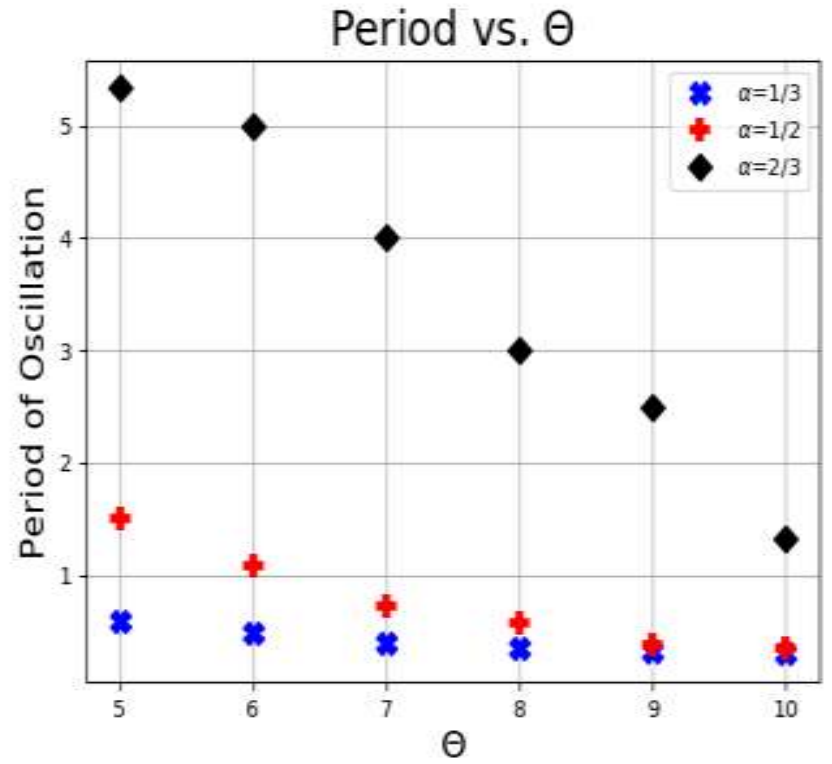

Figure 4.26 The scaled oscillation period vs the thermal expansion ratio $\theta$ for various blockage ratios: $\alpha=1 / 3$ (blue); $\alpha=1 / 2$ (red); and $\alpha=2 / 3$ (black). 


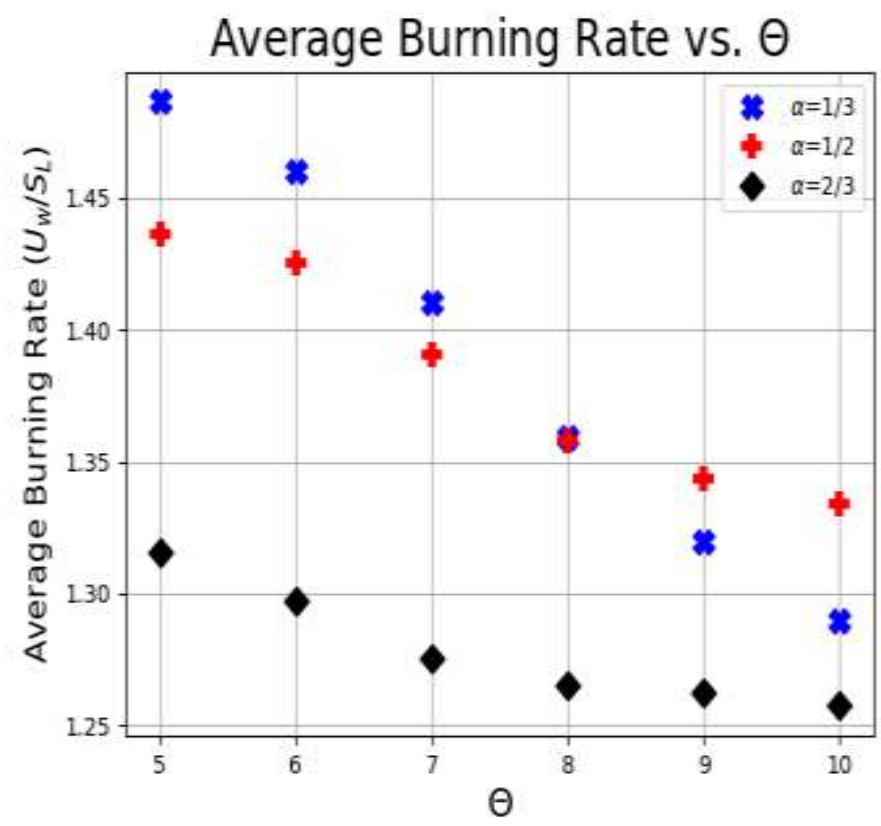

Figure 4.27 The scaled burning rate averaged over an oscillation period vs the thermal expansion ratio $\theta$ for various blockage ratios: $\alpha=1 / 3$ (blue); $\alpha=1 / 2$ (red); and $\alpha=2 / 3$ (black).

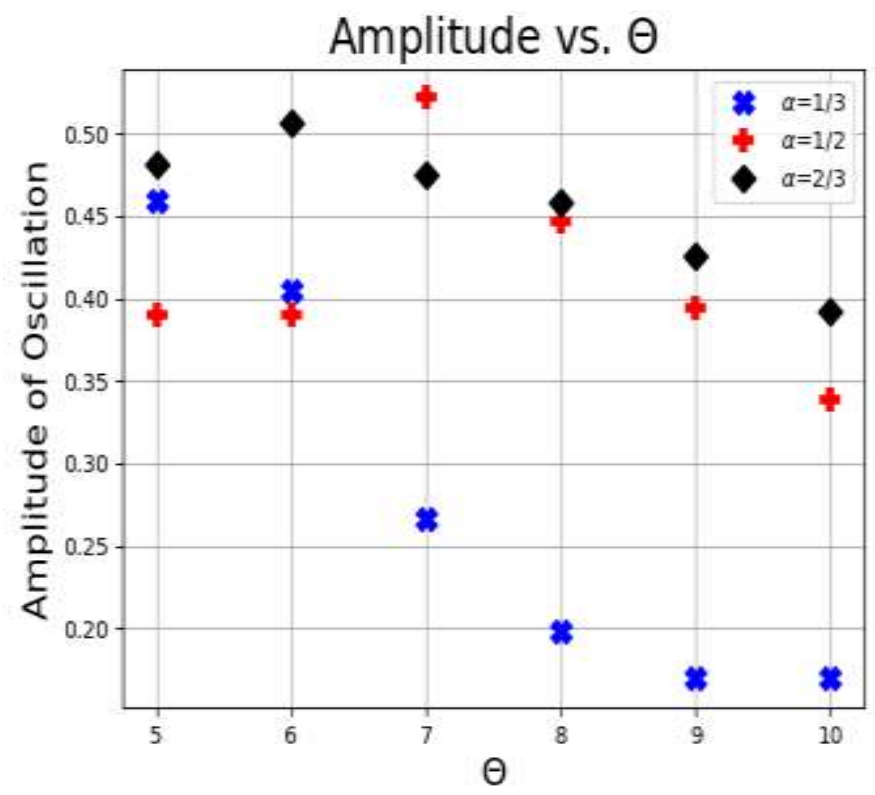

Figure 4.28 The oscillation amplitude vs the thermal expansion ratio $\theta$ for various blockage ratios: $\alpha=1 / 3$ (blue); $\alpha=1 / 2$ (red); and $\alpha=2 / 3$ (black). 

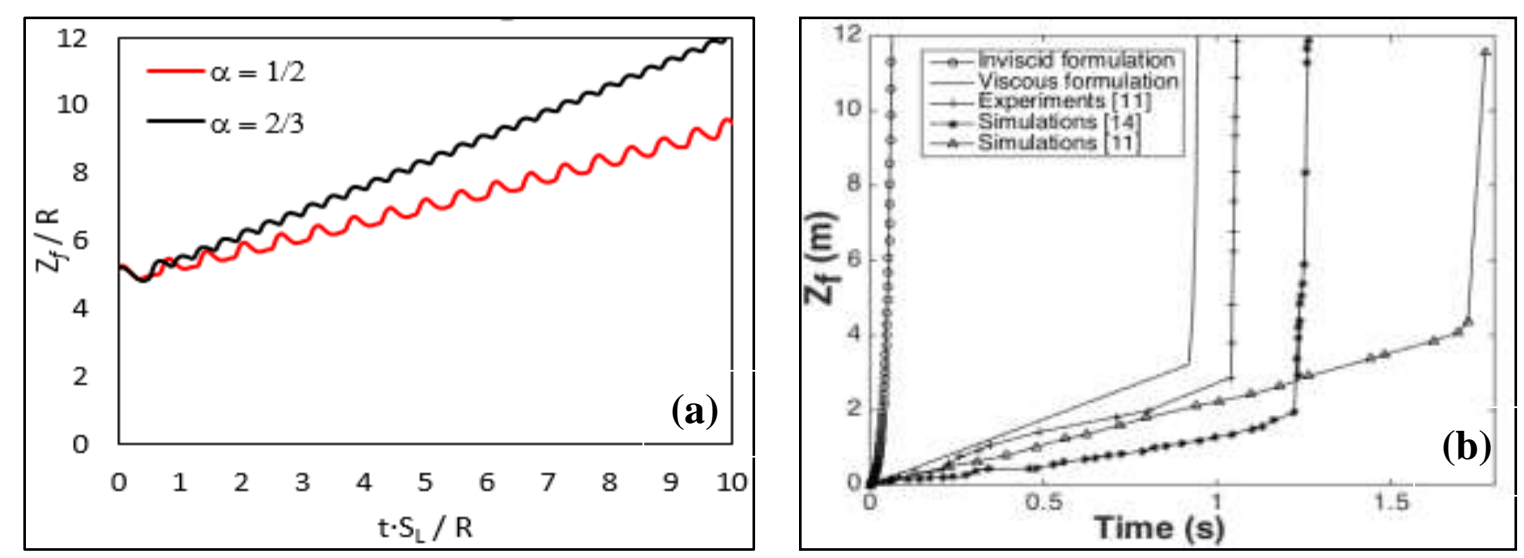

Figure 4.29 The scaled flame position vs the scaled time: a) $R=12 L_{f}, \alpha=1 / 2,2 / 3$ and $\Delta Z / R=$ 1/4. b) recent experiment [24], modeling [23,24] and theory [18].

From Fig. 4.29a the flame oscillations can be treated as fluctuations around a quasi-steady solution. This supports Fig. 4.29b the recent experiments, modeling and theory of flames in obstructed pipes with both ends open, which all yielded steady or quasi-steady flame propagation prior to onset of spontaneous flame acceleration and deflagration-to-detonation transition [12].
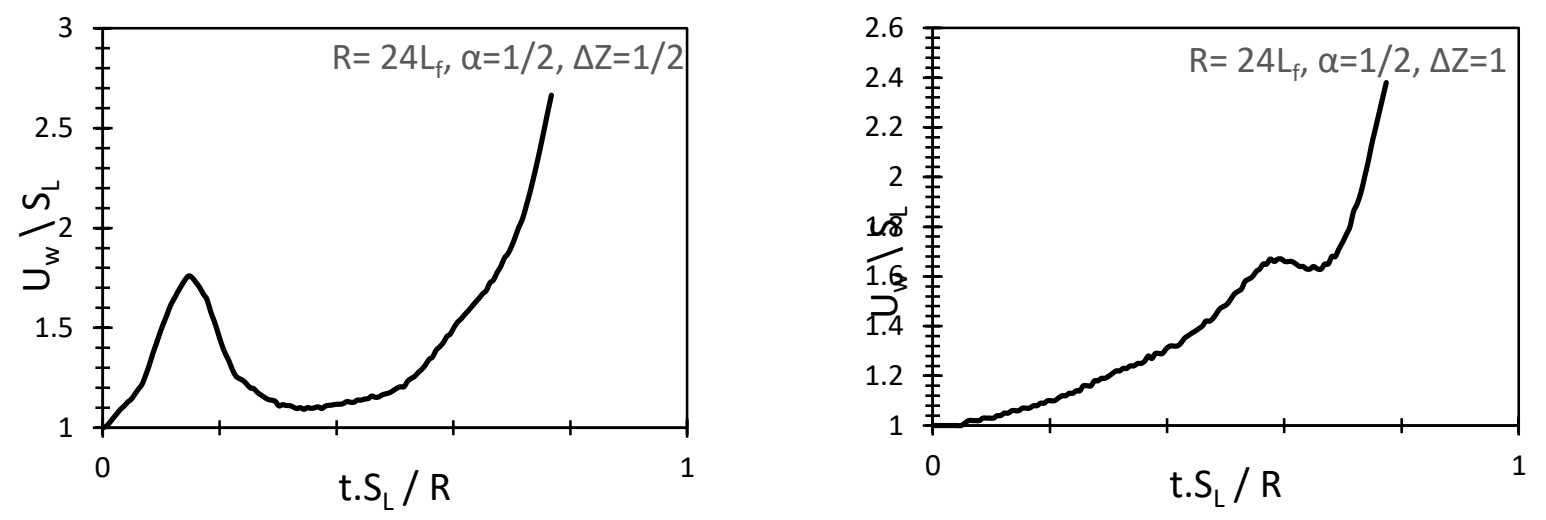

Figure 4.30 The scaled burning rate $U_{t} / S_{L}$ vs the scaled time $\tau$ for $R / L_{f}=24$ and $\Delta Z / R=1 / 2,1$.

Fig. 4.30 is a graph of time evolution against the burning rate of the flame that was plotted to quantify the flame acceleration. Fig. 4.30 represents the flame acceleration for $R=24 L f, \alpha=1 / 2$, $\Delta \mathrm{Z} / \mathrm{R}=1 / 2,1$ respectively. A flame acceleration was noticed when increasing from $\Delta \mathrm{Z} / \mathrm{R}=1 / 4$ to $1 / 2$ and 1 for the $R=24 L_{f}, \alpha=1 / 2$ case. Additionally, in Fig. 4.30 for both $\Delta \mathrm{Z} / \mathrm{R}=1 / 2,1$ it was observed that the flame at some point moved back then propagated again from left to right. This means that there is a possibility of blowback. 

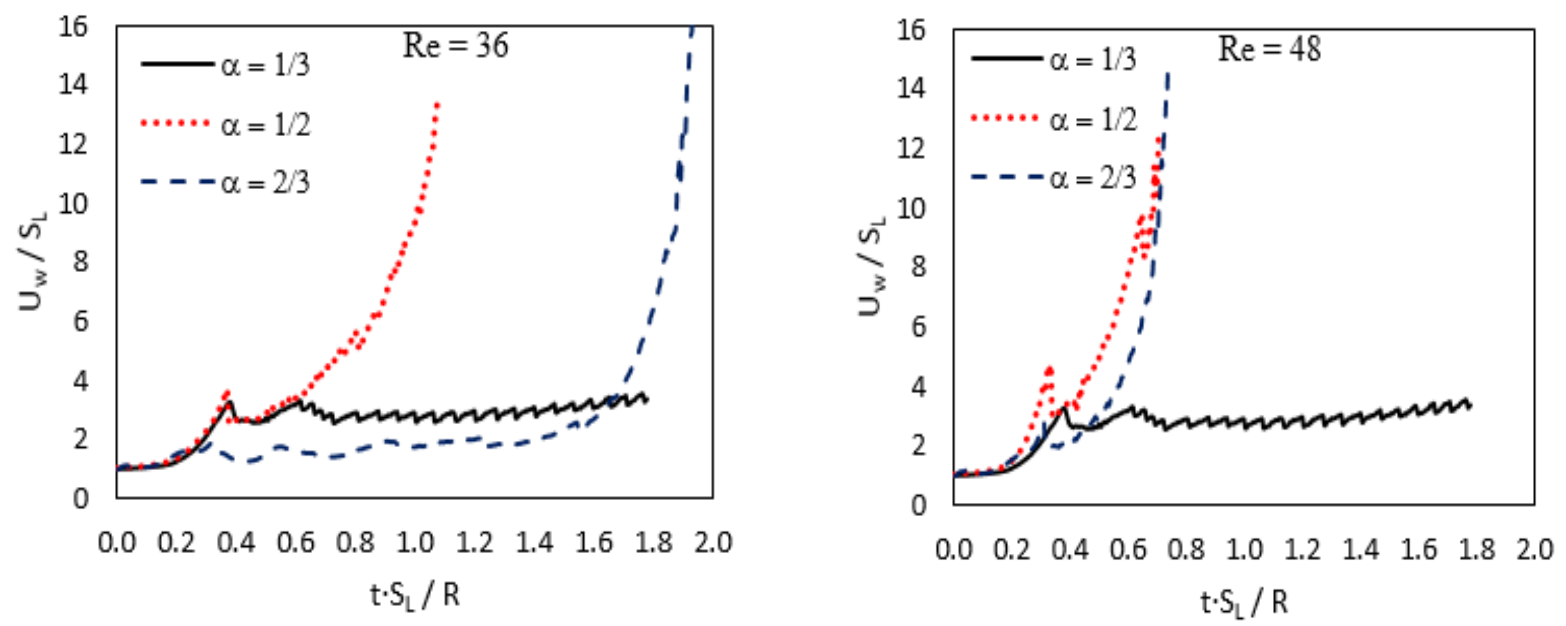

Figure 4.31 The scaled burning rate $U_{t} / S_{L}$ s the scaled time $\tau$ for $R / L_{f}=36,48$ and $\alpha=1 / 2,1 / 3,2 / 3, \Delta Z / R=1 / 4$.

Further considerations were to test what would happen in wider pipes. It was established previously that in relatively narrower pipes the $R / L f=12$, the instantaneous sum of the rate of combustion oscillates each time for the total $\alpha$ taken. The amplitude and the oscillation time differ with $\alpha$ and $R$ and the oscillation observed can be viewed as fluctuation about a quasisteady result. However, it is different case when using wider pipes, $R / L f=36$, 48. Fig. 4.31 shows that there is termination of oscillations as soon as possible, trailed by accelerating flame which proves to be weaker as compared to that in semi-open pipes. 


\section{Chapter 5: Summary}

In the first section, the flame propagation in semi-open obstructed cylindrical pipes was analysed and it was found that an effect of surface friction on flame acceleration is minor, being positive in a pipe with $\Delta Z=R / 2$ and negative for $\Delta Z=R / 4$. Moreover, the surface friction on flame acceleration is minor and can be neglected only when having small obstacle spacing. If the spacing between the obstacles are large and the vorticity is noticeable, then that will break the assumption, and the boundary condition must be considered in this case. On the other hand, the width of the semi-open obstructed channel does not affect the acceleration mechanism. Lastly the blockage ratio which characterizes the flame shape, velocity, propagation manner and the acceleration rate was studied. When blockage ratio is small the flame acceleration halts once it comes into contact with the wall, but if large blockage ratio with large obstacle spacing is present, then that will generate vorticity and vorticity progresses differently with the slip and noslip walls.

It was also illustrated that the effect of the isothermal surfaces as compared to the adiabatic ones is minor, thereby justifying the Bychkov model. Thus, it can be concluded that the real boundary conditions are neither slip nor no-slip; neither adiabatic nor isothermal, but in between these categories.

Although these results can be attributed to the flame dynamics if mainly driven by its spreading in the unobstructed portion of an obstructed pipe, the results, however, are different to those of unobstructed pipes, where the mechanistic and thermal wall status influences the flame dynamics conceptually.

In the second section, analysis was conducted on how premixed flame oscillations and acceleration in 2D obstructed channels with both ends open occur, and it was concluded that the oscillations of the burning rate and non-linearity increased with an increase in $\alpha$.

Now when using channels of half-widths $R / L_{f}=12,24$, blockages ratios $\alpha=1 / 3,1 / 2,2 / 3$ and spacing between the obstacles $\Delta Z / R=1 / 4$, it was concluded that the oscillations resembled those found in unobstructed channels with both ends open [25]. Thus, the oscillations agree with the experiments, modelling and theory of flames in open-open obstructed channels [18, 23, 24]. The 
parameters played significant role on the period and amplitude of the flame oscillation are the blockage ratio, spacing between the obstacles and the thermal expansion. First, it was concluded that the oscillations of the burning rate and non-linearity increased with an increase in $\alpha$. Also, when increasing $\alpha$ the period of oscillations increases. Similar conclusion was achieved for the spacing between the obstacles. The oscillations of the burning rate decrease with an increase in $\Theta$. Furthermore, when $\Theta$ increases the period of the oscillation decreases.

For the case when having half-widths $R / L_{f}=24$, blockage ratio $\alpha=1 / 2$ and spacing between the obstacles $\Delta Z / R=1 / 2$ and 1 it was observed that the flame has initial oscillation then followed by acceleration. Moreover, for this case there is possibility of blowback.

Lastly at wider channels of half-widths $R / L_{f}=36,48$, blockages ratios $\alpha=1 / 3,1 / 2,2 / 3$ and the spacing between the obstacles $\Delta Z / R=1 / 4$, the flame have initial oscillation followed by acceleration. This leads to quasi-steady or steady flame which is propagated past the flame acceleration onset in open-open pipes. 


\section{References:}

1. Putnam A.A. \& Dennis W.C. Organ-pipe oscillations in a flame-filled tube, Proceedings of the Combustion Institute, Vol. 4: 566-574. 1953.

2. Williams F.A. Combustion Theory. Benjamin, Menlo Park, CA. 1985.

3. Oran E. \& Gamezo V. Origins of the deflagration to detonation transition in gas-phase combustion, Combustion and Flame, Vol. 148: 4-47. 2007.

4. Bychkov V. \& Akkerman V. Explosion triggering by an accelerating flame, Physical Review E, Vol. 73: 066305. 2006.

5. Bradley D. Combustion and the design of future engine fuels, Journal of Mechanical Engineering Science, Vol. 223: 2751-2765. 2009.

6. Chiavazzo E., Karlin I., Frouzakis E. \& Boulouchos K. Method of invariant grid for model reduction of hydrogen combustion, Proceedings of the Combustion Institute, Vol. 32: 519-526. 2009.

7. Valiev D., Bychkov V., Akkerman V., Law C.K. \& Eriksson L.-E. Flame acceleration in channels with obstacles in the deflagration-to-detonation transition, Combustion and Flame, Vol. 157: 1012-1021. 2010.

8. Demir S., Bychkov V., Chalagalla S.H.R. \& Akkerman V. Towards a predictive scenario of a burning accident in a mining passage, Combustion Theory and Modelling, Vol. 21: 997-1022. 2017.

9. Demirgok B \& Akkerman V. Theory of flame acceleration in tubes due to wall friction: Intrinsic limitations and accuracy, Paper \#3D01, 8th US National Combustion Meeting, Park City, UT, USA, May 19-22, 2013.

10. Canuto A.Q. Fundamentals of Fluid Dynamics, Springer. 2007.

11. Schönfeld T.P. Influence of boundary conditions in LES. CTR Briefs. 1999.

12. Akkerman V., Bychkov V., Petchenko A. \& Eriksson L.-E. Accelerating flames in cylindrical tubes with nonslip at the walls, Combustion and Flame, Vol. 145: 206-219. 2006.

13. Bychkov V., Petchenko, A., Akkerman, V. \& Eriksson L.-E. Theory and modeling of accelerating flames in tubes, Physical Review E, Vol. 72: 046307. 2005. 
14. Johansen C., Kellenberger M., Pinos T. \& Ciccarelli G. Flame acceleration in narrow channels with obstacles, The 2012 Conference of the Computational Fluid Dynamics Society of Canada (CFD2012), Canmore, CA, May 9-11, 2012.

15. Bychkov V., Valiev D. \& Eriksson L.E. Physical mechanism of ultrafast flame acceleration, Physical Review Letters, Vol. 101: 164501. 2008.

16. Bychkov V., Akkerman V., Fru G., Petchenko A \& Eriksson L.E. Flame acceleration at the early stages of burning in tubes. Combustion and Flame, Vol. 150: 263-276. 2007.

17. Ugarte O.J., Bychkov V., Sadek J., Valiev, D. \& Akkerman V. Critical role of blockage ratio for flame acceleration in channels with tightly spaced obstacles, Physics of Fluids, Vol. 28: 093602. 2016.

18. Bychkov V., Sadek J. \& Akkerman V. Analysis of flame acceleration in open or vented obstructed pipes, Physical Review E, Vol. 95: 013111. 2017.

19. Zeldovich Y.B., Barenblatt G., Librovich V. \& Makhviladze G. The Mathematical Theory of Combustion and Explosions, New York: Consultants Bureau. 1985.

20. Law C.K. Combustion Physics, Cambridge University Press, New York, 2006.

21. Valiev D., Akkerman V., Kuznetsov M., Eriksson L.-E., Law C.K. \& Bychkov V. Influence of gas compression on flame acceleration in the early stage of burning in tubes, Combustion and Flame, Vol. 160: 97-111. 2013.

22. Wollblad C., Davidson L. \& Eriksson L.-E. Large eddy simulation of transonic flow with shock wave / turbulent boundary layer interaction, AIAA Journal, Vol. 22: 2340-2353. 2006.

23. Middha P. \& Hansen O.R. Predicting deflagration to detonation transition in hydrogen explosions. Process Safety Progress Vol. 27, 192-204 2008.

24. Yanez J. Kuznetsov M. \& Bykov V. Sudden acceleration of flames in open channels driven by hydraulic resistance, The $24^{\text {th }}$ International Colloquium on the Dynamics of Explosions and Reactive Systems (ICDERS), Taipei, Taiwan, July 28-August 2, 2013, paper \#164, pp. 1-6.

25. Akkerman V., Bychkov V., Petchenko A. \& Eriksson L.-E. Flame oscillations in tubes with nonslip at the walls, Combustion and Flame, Vol. 145: 675-687. 2006. 